Chapman University

Chapman University Digital Commons

ESI Working Papers

Economic Science Institute

$9-23-2020$

\title{
An Elementary Humanomics Approach to Boundedly Rational Quadratic Models
}

\author{
Michael J. Campbell \\ Vernon L. Smith \\ Chapman University, vsmith@chapman.edu
}

Follow this and additional works at: https://digitalcommons.chapman.edu/esi_working_papers

Part of the Econometrics Commons, Economic Theory Commons, and the Other Economics Commons

\section{Recommended Citation}

Campbell, M. J., \& Smith, V. L. (2020). An elementary humanomics approach to boundedly rational quadratic models. ESI Working Paper 20-35. https://digitalcommons.chapman.edu/esi_working_papers/ $330 /$

This Article is brought to you for free and open access by the Economic Science Institute at Chapman University Digital Commons. It has been accepted for inclusion in ESI Working Papers by an authorized administrator of Chapman University Digital Commons. For more information, please contact laughtin@chapman.edu. 


\section{An Elementary Humanomics Approach to Boundedly Rational Quadratic Models}

Comments

ESI Working Paper 20-35

This paper later underwent peer review and was published as:

Campbell, M. J., \& Smith, V. L. (2020). An elementary humanomics approach to boundedly rational quadratic models. Physica A, 562, 125309. https://doi.org/10.1016/j.physa.2020.125309 


\title{
An Elementary Humanomics Approach to Boundedly Rational Quadratic Models
}

\author{
Michael J. Campbell ${ }^{*}$ \\ Eureka, USA \\ Vernon L. Smith** \\ Chapman University
}

\begin{abstract}
We take a refreshing new look at boundedly rational quadratic models in economics using some elementary modeling of the principles put forward in the book Humanomics by Vernon L. Smith and Bart J. Wilson. A simple model is introduced built on the fundamental Humanomics principles of gratitude/resentment felt and the corresponding action responses of reward/punishment in the form of higher/lower payoff transfers. There are two timescales: one for strictly self-interested action, as in economic equilibrium, and another governed by feelings of gratitude/resentment. One of three timescale scenarios is investigated: one where gratitude/resentment changes much more slowly than economic equilibrium ("quenched model"). Another model, in which economic equilibrium occurs over a much slower time than gratitude/resentment evolution ("annealed" model) is set up, but not investigated. The quenched model with homogeneous interactions turns out to be a non-frustrated spin-glass model. A twoagent quenched model with heterogeneous aligning (ferromagnetic) interactions is analyzed and yields new insights into the critical quenched probability $p(1-p)$ that represents the empirical frequency of opportunity for agent $i$ to take action for the benefit (hurt) of other that invokes mutual gratitude (resentment). A critical quenched probability $p_{i}^{*}, i=1,2$, exists for each agent. When $p<p_{i}^{*}$, agent $i$ will choose action in their self-interest. When $p>p_{i}^{*}$, agent $i$ will take action sensitive to their interpersonal feelings of gratitude/resentment and thus reward/punish the initiating benefit/hurt. We find that the $p_{i}^{*}$ are greater than one-half, which implies agents are averse to resentful behavior and punishment. This was not built into the model, but is a result of its properties, and consistent with Axiom 4 in Humanomics about the asymmetry of gratitude and resentment. Furthermore, the agent who receives less payoff is more averse to resentful behavior; i.e., has a higher critical quenched probability. For this particular model, the Nash equilibrium has no predictive power of Humanomics properties since the rewards are the same for self-interested behavior, resentful behavior, and gratitude behavior. Accordingly, we see that the boundedly rational Gibbs equilibrium does indeed lead to richer properties.
\end{abstract}

Keywords: Agent Based Model, Bounded Rationality, Correlation Inequality, Humanomics, Phase Transition, Potential Game, Spin Glass 


\section{Introduction}

The analysis of economic interactions in the book Humanomics [1] shows us that the standard economic models of self-interested action can often lead to incorrect predictions. Indeed, Humanomics outlines how experimental economics yielded evidence in two-person games, such as the trust and ultimatum games, that falsified predictions in the 1980s and 1990s, and it wasn't until the 2000s that resolutions began to appear. What fundamentally needs to be included in the models are considerations that account for human sentiment and sociability. Adam Smith does this in [2], using the building blocks of sentiments, fellow-feeling/empathy, and a sense of propriety. Such considerations run much deeper than the maximum utility techniques ('Max U') previously used, which lead to incorrect predictions in the laboratory [1]. We propose to implement some of these building blocks at an elementary level, as a first-attempt to examine their effects on economic or social systems of arbitrary size - small or large. It is pointed out that interactions in a large economy are less based on rules of sociability and more based on the mutual benefit of efficient exchange [3]. ${ }^{1}$

Adam Smith's model of human sociability involves inherently small numbers. Parents, siblings, uncles, aunts, cousins, extended family in the form of neighbors and friends. In fact, the population size $N$ considered by the model is largely limited by the knowledge state that it is possible for individuals to have of each other. People are all self-interested, but are motivated to act in other-regarding ways driven by the calculus of benefit-hurt or reward-punishment based on common knowledge that all are self-interested and thus benefit if they get more of something beneficial, and are hurt if they get less (individual 'non-satiation'). Socially bonded people desire praise/praiseworthiness and achieve it through rule-following gratitude/reward social exchange; and to avoid blame/blameworthiness which is achieved by avoiding deliberately hurtful actions toward others. This leads to efficient social outcomes.

In the economy, orders of magnitude larger, people are also self-interested and act in their self-interest. What binds people into a system is not benefit/hurt rule-following based on actions that are social signals, but action based on prices, where prices coordinate people unknown to each other to take actions that benefit themselves and others through gains from exchange. Because all exchange involves gains for both parties, and incentivizes specialization, the outcome is efficient and wealth-enhancing.

Here, we examine the effects of the sentiments of gratitude and resentment, invoked by intentional actions that benefit or hurt other and lead to reciprocal acts of reward and punishment. Actions may invoke either gratitude or resentment. If one does not know what another resents, one can't know what actions to avoid. Therefore, it is acceptable for there to be punishment to convey the sentiment of resentment, just as reward conveys gratitude.

For the sake of simplicity for this initial model, we consider reward to be an increase in payoff and punishment to be a decrease in payoff. This follows the basic premise of non-satiation, while keeping the model as simple as possible. In order for agents to enact rewards and punishment

\footnotetext{
${ }^{*}$ Corresponding author.

** Research supported by Chapman University, Economic Science Institute.

Email addresses: michaeljcampbell@outlook.com (Michael J. Campbell), vlomaxsmith@gmail.com (Vernon L. Smith)

${ }^{1}$ This makes sense since a buyer may never interact with a seller again when there are a large number of sellers. A buyer could 'burn their bridge' with the seller, by leaving an unjustified bad review online, etc., and then never interacting with the seller again to avoid repercussions of bad feelings. A person would be much less likely to do that with a friend or regular acquaintance such as a coworker, since they see each other on a regular basis.
} 
in this manner, they need to have knowledge whether a particular action, or change in strategy, results in an increase or decrease in the payoff of another agent. In Adam Smith's Theory of Moral Sentiments [2], all utility functions are strictly self-interested and independent. That is how one knows what benefits (or hurts) another; and it must be common knowledge so that every action can unambiguously be known to all others as benefit or hurt.

In this work, we use action functions (as distinct from utility functions) which enable individuals to enact rewards or punishments that implement the rules of sociability. In our model, we take the perspective that agents themselves are altering their self-interested actions depending on their gratitude and resentment configuration. It is through this gratitude configuration that the action function changes. Such action functions better reflect Adam Smith's fundamental assumption that all are strictly self-interested, but agents' actions may be other as well as own regarding. Smith's propositions govern rule-following actions by strictly self-interested actors, but those rules involve transfers that end up augmenting payoffs and utility. In [1] (page 27), it is mentioned: "our question is whether augmenting a utility function, parsimoniously or not, explains the friend's choice, or merely assumes that which is to be explained." We contrast that with the treatment of action functions in this work, where agents add or subtract functions from their own action function to enact reward or punishment based on their current state of gratitude or resentment towards other agents. We do not attempt to explain agents' behavior, but rather we use action functions to enact Adam Smith's fundamental principles on behavior and action. We are assuming that agents should be able to alter their behavior from payoff equilibrium to reward or punish other agents. We do not assume the agents take any particular course of action for reward or punishment, as this is based on a probability measure (i.e., the gratitude configuration, which is a collection of random variables in this elementary treatment). Finally, we construct the action functions so that when agents maximize their action function, they are self-interested (want to increase payoff), yet are able to realize the aforementioned transfers to other agents to enact reward or punishment.

The spirit of this paper is that agents experiment with their behavior (which is why we use bounded rationality) to discover what behavior results in reward or punishment. After learning this, agents use these techniques to reward or punish other agents. The model here starts at this point: agents are assumed to know how to control their behavior to realize actions of reward or punishment through the alteration of their optimizing behavior - i.e., the alteration of action functions. $^{2}$ The addition or subtraction of payoffs or other functions to action functions in our model is a mathematical representation of agents altering their behavior. Indeed, for an agent to alter their behavior to something other than equilibrium for a payoff function, the agent would have to alter their decision/strategy variable. Thus, agents have a "control" to alter their behavior to achieve desired ends of rewarding or punishing other agents. In fact, the process of an agent altering the dynamics of their decisions (by changing what it optimized) can be interpreted as a problem in control theory (see [4] or the topic of "mean-field games" where the agents "control" their dynamics in the game).

Another recurring critique of utility function representations is that they depend only on outcomes ([1] pages 27,40 ). The action functions in this work depend on a gratitude configuration, which is determined by the feelings and sentiments of agents, and a function that agents choose to add (we limit that to positive or negative payoffs here for tractability and simplicity). Furthermore, as a result of this approach, the action functions are not static, but change depending

\footnotetext{
${ }^{2}$ For one-shot games where agents do not know the effects of adding functions to their action function, this model would have to be modified. Our treatment in this paper is for long-term behavior.
} 
on the sentiments of agents. It is mentioned in [1] (page 79) with motivation from a statement from Adam Smith himself ([2] First.I.II, page 10): "Both the pleasure and the pain are always felt so instantaneously, and often upon such frivolous occasions, that it seems evident that neither of them can be derived from any such self-interested considerations", i.e. utility functions. In our (elementary) model, pleasure or pain is determined by an increase or decrease of pay$o f f$, respectively - i.e., an action, which is consistent with Adam Smith's foundational view and non-satiation. For example, in terms of the economic system:

- the alteration of the action function of agent $i, a_{i}$, results in a change of payoff to agent $j$;

- if that alteration increases (decreases) agent $j$ 's payoff, then it is regarded by agent $i$ and $j$ as a benefit (hurt) and we refer to $j$ as feeling gratitude (resentment) toward $i$;

- now if agent $j$ acts in reciprocal response towards agent $i$, and $i$ approves (condemns) $j$ 's action, then $i$ will reward (punish) agent $j$ by altering $a_{i}$ to yield an increase (decrease) in j's payoff.

The intention is to incorporate the observations Smith mentions into some existing models in an elementary, tractable way. We limit the model so that many existing tools and techniques can be used, but we encourage the extension of these models to realize the goals and objectives in Humanomics [1] in a more sophisticated manner.

\section{History of the Model}

Quadratic models originate as far back as 1838 in a Cournot competition with linear price [5]. More recently, variations on a Cournot competition with localized demand were studied in [6], and a quadratic game-theoretic model of financial interactions among a hedging agent and speculators was examined in $[7,8,9,10,11]$. The work [12] contains various applications of quadratic models: crime, education, industrial organization, cities, conformity and conspicuous effects.

\section{Other Works}

The approach used for the models presented here is to use an existing economic model augmented by principles from Humanomics, and then to superimpose a mechanism for bounded rationality onto the model. It is important to point out that the essential underlying economic model is unchanged, which is evident from the fact that the models and their boundedly rational extensions here are both potential games and make extensive use of the potential. As a result, artifacts from physics such as an "external field" (linear payoff/action terms), "energy" (negative of the potential), "susceptibility" (spatial volatility), "temperature" (temporal volatility), etc., result from reinterpreting components of the economic theory; not vice-versa. In the boundedly rational case, the potential is used for the dynamics (the drift), which results in these associations.

In [13], a dynamic model based on the Fokker-Planck equation is used to model sociological phenomena and interactions, such as opinion formation and population dynamics. The construction of the Fokker-Planck equation is not based on pre-existing tools from economics, but rather on first-principles that are justified from the ground-level up in the book. Others have studied models of agent behavior in microeconomic theories containing randomness, as is done in the current work. In [14], such bounded rationality was analyzed. The existence of a potential in 
the current work leads to the Gibbs equilibrium measure ${ }^{3}$ and thus is fully integrated into the realm of statistical mechanics. Until [15], twelve years after [6], no other works had connected potential games to Gibbs equilibrium measures, although some had come close [16, 17], [18], [19]. Others have used mean-field Curie-Weiss models to model socio-economic interactions [20]. These models are constructed a-priori and are not the result of beginning with a potential game (although this could be done). In fact, any model that uses a Gibbs measure for a probability distribution (mean-field or otherwise) can be interpreted as a boundedly rational potential game.

\section{Model}

We consider a game with a finite number $N$ of "agents". All of these agents belong to the set $\Lambda$. At any moment in time, an agent $i \in \Lambda$ can select a strategy $x_{i} \in F_{(i)}$ and the $x_{i}$ are the strategy variables. A configuration $\mathbf{x}$ of the system is any possible state of the system:

$$
\mathbf{x}=\left(x_{1}, x_{2}, \ldots, x_{N}\right),
$$

where each $x_{i} \in F_{(i)}$. The set of all possible configurations of the game is

$$
\Phi_{\Lambda}:=\prod_{i \in \Lambda} F_{(i)},
$$

which is called (pure) state space. The $F_{(i)}:=F$ here is the set

$$
F:=[\underline{x}, \bar{x}],
$$

with $\underline{x}<\bar{x}$. For example, $\underline{x}$ and $\bar{x}$ could be a lower bound and upper bound, respectively, on the number of goods produced in a Cournot competition.

Now we will denote the action function of each agent as

$$
a_{i}(\mathbf{x})
$$

\subsection{Humanomics Implementation}

We emphasize that the content of this section constitutes a primitive implementation of the principles in Humanomics [1]. We take this approach as a starting point, since several discussions [3] have elucidated the authors to the complexity of these ideas. As such, we take an incremental approach, starting with the most elementary ideas, and then building on those subsequently.

An important principle in [1] is Adam Smith's Third Axiom ([2] Second.I.I page 94):

The sentiment which most directly and immediately prompts us to reward, is gratitude; that which most immediately and directly prompts us to punish, is resentment.

\footnotetext{
${ }^{3}$ The Gibbs measure is the stationary state of stochastic dynamics of strategy variables, and the stochastic dynamics implement bounded rationality. In this work, by the phrase economic equilibrium we mean that enough time has elapsed in the stochastic dynamics so that the strategy variables are Gibbs-distributed; i.e., the stationary state has been reached by the dynamics.
} 
Gratitude and resentment are a result of actions of others on ourselves or someone else ([1] page 24, last paragraph). The sentiments of gratitude and resentment ([1] pages 23-24, third bullet) can arise as a result of empathy, or "fellow feeling" (page 24). With this in mind, we assign a payoff function to each agent, which we denote

$$
\pi_{i}(\mathbf{x})
$$

which yields a payoff to agent $i$ given the configuration $\mathbf{x}$. This function must capture transfers from agent $i$ to agent $j$ and returns from agent $i$ to agent $j$ in accordance with agent $i$ 's benefit from agent $j$ 's action, and agent $i$ 's reward to agent $j$. In general, there are gains from these exchanges, so both are made better off exactly as with economic exchange, but the transfers are in-kind, not monetary. Also, they are not simultaneous. Because of a relationship between agent $i$ and agent $j$ there is a fellow-feeling, whereby if agent $i$ takes action to transfer goods from agent $i$ to agent $j$, agent $j$ feels an obligation to transfer goods from agent $j$ to agent $i{ }^{4}$ The same holds for hurtful actions if agent $i$ transfers away from agent $j$ to agent $i$, (e.g., theft). The reward/punishment response actions are helpful when there is less than perfect information. ${ }^{5}$ Punishment out of resentment is a way of conveying that agent $j$ did not like agent $i$ 's action toward agent $j .{ }^{6}$

Gratitude, resentment, and self-interest among agents will be modeled with variables that affect payoffs. We denote the gratitude configuration $\gamma$ as

$$
\gamma(i, j)=\gamma_{i j},
$$

where $\gamma_{i j}$ is a real number. Note that $\gamma_{i j}$ represents the gratitude agent $i$ has for agent $j$, and that $\gamma_{i j}$ need not equal $\gamma_{j i}$. Agent $i$ and agent $j$ are pre-connected in a way that allows agent $i$ to feel gratitude or resentment from an action by agent $j$, and vice-versa. If there is no action, there is no gratitude or resentment. Moreover, the gratitude or resentment felt is proportioned to the amount transferred by the action. We can visualize the agents to be vertices on a graph, and the bond configuration to be directed bonds between vertices. For example, $\gamma_{i j}$ would be represented by a bond directed out of vertex $i$ into vertex $j$. Any two vertices would then have two connecting bonds representing the gratitude each agent has for the other.

Example 1 (Gratitude Configuration with Conditional Agent-Exchange Symmetry). A gratitude configuration that has agent-exchange symmetry when conditioned on gratitude or resentment has the form

$$
\gamma(i, j)=\gamma_{i j}= \begin{cases}w_{g} & \text { i has gratitude for } j \\ w_{r} & \text { i resents } j\end{cases}
$$

\footnotetext{
${ }^{4}$ For example, I bring your trash barrel in this week, and you bring in mine next week. Such reciprocal actions are efficiency enhancing, and create and grow social wealth. These transfers always meet with third party approval via network consensus; bilateralism becomes pluralism.

${ }^{5}$ I don't know the value to you of a favor I do you, and you don't know the value to me of your reciprocal reward It is not like knowing the exact payoffs to each for every action as in the single-play trust games. In this sense it is like gains from an economic exchange. I pay you $\$ 800$ for an Iphone. You don't know it, but my reservation price was $\$ 1200$, and I don't know that yours was $\$ 100$ ! In both cases we know only that there is a perceived gain which is sufficient for motivation - "to bring it thither" as Adam Smith would say.

${ }^{6}$ In rule-of-law civil government societies, actions under the gratitude-reward calculus are adopted in the form of trade, where the transfers are positive, but they are either simultaneous, or a credit system assures goods are returned, and third party enforcement of exchanges as contracts extends to strangers allowing specialization and trade to grow wealth over much larger populations. On the negative side the calculus of resentment and punishment becomes property, prohibiting murder, theft/robbery and violation of contract under penalties proportionate to the harm. Hence, property, by punishing involuntary transfers, supports exchange.
} 
where $w_{g}$ and $w_{r}$ are weights for mutual gratitude and mutual resentment, respectively. The weights $w_{g}\left(w_{r}\right)$ are realized only with a positive benefit (negative loss). Note that we are making a simplifying assumption that the weights $w_{g}$ and $w_{r}$ have agent-exchange symmetry. For example, if agent $i$ resents agent $j$ then the weight for that resentment is the same as the weight of resentment when agent $j$ resents agent $i$, or mathematically, $\gamma_{i j}=\gamma_{j i}$ if $i$ resents $j$ and $j$ resents $i$.

The possible bond pairs between $i$ and $j$ are $\left(\gamma_{i j}, \gamma_{j i}\right)$, which can be $\left(w_{g}, w_{g}\right),\left(w_{g}, w_{r}\right)$, $\left(w_{r}, w_{g}\right)$, and $\left(w_{r}, w_{r}\right)$. A variation would be the inclusion of a self-interested weight $w_{s}$ which would not be considered gratitude nor resentment.

Example 2 (Gratitude Configuration with Full Agent-Exchange Symmetry). A gratitude configuration with full agent-exchange symmetry is when $\gamma_{i j}=\gamma_{j i}$. Then we visualize a gratitude configuration as a set of single undirected bonds between each pair of vertices $i$ and $j$, and this bond represents the mutual gratitude or mutual resentment, with weight $\gamma_{i j}=\gamma_{j i}$. Note in this case it is not simultaneously possible for agent $i$ to have gratitude for agent $j$, and agent $j$ to resent agent $i$. This is a reasonable simple model, as [1] mentions how good and bad behavior is reciprocated (page 39; page 86 Corollary) and how sentiments tend to align (page 47). Either agent can feel gratitude or resentment, depending whether an action conveyed benefit or hurt.

If the values for $\gamma_{i j}$ are $w_{g}$ for mutual gratitude, and $w_{r}$ for mutual resentment, then the single bond between $i$ and $j$ can be represented as $\left(w_{g}\right)$ or $\left(w_{r}\right)$.

We will create action functions that implement Axiom 3 of [1] (page 71), which states that gratitude and resentment prompt agents to reward or punish other agents. We will use the effect agent $i$ can have on the payoffs of other agents to implement this idea. Let the action of agent $i$ be given by

$$
a_{i}=\sum_{j \in \Lambda} \gamma_{i j} \pi_{j}
$$

where $\gamma_{i i}=1$. Note that this action has a purely self-interested part, $\pi_{i}$, as mentioned in the general formulation in [1] (e.g., equation (3) in chapter 11). The gratitude configuration and presence of other's payoffs incorporates the same ideas of praised/praiseworthy actions in that general formulation, albeit in a different, simpler form.

It is further mentioned in [1] (page 24 and page 73 axiom 4) that gratitude and resentment are asymmetrical, and that resentment is more influential, and carries more weight than gratitude. Accordingly, $w_{r}$ in Example 1 and Example 2 should result in a larger negative effect than the positive effect induced by $w_{g}$.

We again emphasize that this approach does not capture the full depth of the ideas in [1]. The examples below in Subsection 4.2 show these limitations ${ }^{7}$. Consider the example in [1] about envy ("A Theory of Enviousness", page 50), and those about trust games (Chapter 8 page 117, specifically the comment "... knowledge of the payoffs affects play in the trust game"). It is clear, for games involving a small state space and a small number of players, that players implement rewards and punishments through their knowledge of the payoffs of the games, by choosing strategies that result in a higher or lower payoff for the other agent(s). We regard different choices

\footnotetext{
${ }^{7}$ See, for example, comments in [1] (page 40, second paragraph) about maximum utility alone being inadequate for personal, social interactions because such an approach only considers outcomes, and not intentions, which can result in the selection of non-Nash-equilibrium outcomes. In staying with the spirit of simplicity, we will put forward a "naive" remedy to this by implementing bounded rationality, which will result in a probability applied to all outcomes and have perturbations to action optimization.
} 
as transferring (or not) different known amounts. This is operationally distinct from the gratitude configuration approach above that alters action functions. However, these action functions have the same effect of transferring (or not) amounts to (or from) other agents. Furthermore, the agents may choose states that are not Nash equilibrium states in order to implement reward and punishment.

From the observations in [1], for more complex games having more agents and/or more complex payoffs, one reasonable model is to assume agents myopically explore different strategies and observe the resulting payoffs at each step of the game. The agents then develop heuristics that they follow to implement reward and punishment of other agents. If agents were playing a boundedly rational quadratic potential game with positive coefficients (aligning case; "ferromagnetic" case in physical systems), and all payoffs have positive coefficients, they would learn that payoffs are positively correlated [21] and could use the mechanism of gratitude configurations in (8) to predictably reward and punish other agents. This will be shown to break down for negative coefficients (opposing case; "antiferromagnetic" case in physical systems) below in Example 6.

The reason we investigate gratitude configurations is that they are more amenable to the computational analysis in this work. Furthermore, when the economics system considered here has a large number of constituents, it is not realistic to expect agents to have knowledge of the intricacies of the effects of the payoffs on all agents in the system, since the amount of computation to do so is infeasible for a single agent in a very large system. ${ }^{89}$

As a result of this, we will consider the effects of bounded rationality on decisions and assume the gratitude configurations fluctuate with a random distribution relative the equilibration of the agents. This bounded rationality is an attempt to capture that agents do not necessarily play the Nash equilibrium, and can play different strategies depending on the desire to reward, punish, or remain indifferent. For simplicity, we avoid modeling the detailed dynamics of such behavior on strategies and gratitude configurations, but rather capture the more general idea that agents should play different pure strategies with various gratitude configurations with a given probability distribution.

\subsection{Quadratic Models}

General quadratic models have payoffs that are quadratic forms in the strategy variables:

$$
\pi_{i}(\mathbf{x})=\sum_{k \geq j=1}^{N} J_{j k}^{(i)} x_{j} x_{k}+\sum_{j=1}^{N} h_{j}^{(i)} x_{j}+C_{i},
$$

where $\pi_{i}$ is the payoff to agent $i, J_{j k}^{(i)}$ and $h_{j}^{(i)}$ are real numbers, and $C_{i}$ is a constant. Note that the coefficients $J_{i j}$ are only defined for $i \leq j$, so we may assume that $J_{i j}=0$ when $i>j$.

\footnotetext{
${ }^{8}$ Alternately, we can assume bounded rationality occurs as a result of human beings being irrational in some situations. As the pandemic of 2020 has shown in many parts of the United States, people have unnecessarily been hoarding certain supplies, such as toilet paper, despite the fact that supply of these products, which satiated demand in the past, had not gone down.

${ }^{9}$ Yet another way to interpret bounded rationality is for non-human agents, such as artificial intelligence decision makers. The dynamics used subsequently are those of 'reinforcement learning' (RL), and an artificial decision maker can use these dynamics. These artificial agents use 'training' data to construct an estimator, such as an RL process or a neural network, that makes decisions. It is a common technique to introduce error in the training process, which increases the bias of the estimator, but reduces the variance, to prevent 'overfitting'. Because of this increased bias, there will be inherent error in the decisions. One of the authors was informed about such AI being implemented in financial trading models by a 'quant' friend [22].
} 
We point out that adding and/or subtracting quadratic payoffs results in another quadratic payoff. This is a nice property of the gratitude configuration approach to actions in (8).

\subsection{Potential Games}

A potential game [23] with potential $V(\mathbf{x})$ and action functions $a_{i}(\mathbf{x})$, for each agent $i \in \Lambda$ satisfies, by definition,

$$
\frac{\partial}{\partial x_{i}} a_{i}(\mathbf{x})=\frac{\partial}{\partial x_{i}} V(\mathbf{x}) .
$$

The salient point is that, for each $i$, the gradient of the potential with respect to the variables of agent $i$ is the same as the gradient of the $i$ th agent's action function (with respect the $i$ th agent's variables). In a dynamical interpretation, agents would follow the gradient of their action function for "myopic decisions" (agents look at the best local choice). For potentials with an interior maximum, this would lead to the Nash equilibrium [23].

For the actions in (8) to be those of a (necessarily quadratic) potential game, the necessary conditions in (10) on the quadratic payoff in (9) imply that

$$
\frac{\partial^{2}}{\partial x_{j} \partial x_{i}} a_{i}(\mathbf{x})=\frac{\partial^{2}}{\partial x_{i} \partial x_{j}} a_{j}(\mathbf{x}) .
$$

Without loss of generality, assume $i<j$. The conditions in (11) imply that the coefficients in (9) satisfy

$$
\sum_{k} \gamma_{i k} J_{i j}^{(k)}=\sum_{k} \gamma_{j k} J_{i j}^{(k)}, \quad i<j
$$

The quadratic function

$$
V=\sum_{j \geq i=1}^{N} K_{i j} x_{i} x_{j}+\sum_{i=1}^{N} h_{i} x_{i}
$$

will be a potential for the payoffs in (9) if (10) holds. This results in the following conditions for a potential:

$$
\begin{aligned}
K_{i i} & =\sum_{k} \gamma_{i k} J_{i i}^{(k)}, & 1 & \leq i \leq N, \\
K_{i j} & =\sum_{k} \gamma_{i k} J_{i j}^{(k)}, & 1 & \leq i<j \leq N, \\
K_{j i} & =\sum_{k} \gamma_{i k} J_{j i}^{(k)}, & 1 & \leq j<i \leq N, \\
h_{i} & =\sum_{k} \gamma_{i k} h_{i}^{(k)}, & 1 & \leq i \leq N .
\end{aligned}
$$

Note that (14b) and (14c) should be independent of the particular index $i$ occurring in the first or second position. For example, consider the equation for the particular case $K_{15}$. We can consider $i=1$ or $i=5$, in which case the two equations for those values from (14b) and (14c) are

$$
\begin{aligned}
K_{15} & =\sum_{k} \gamma_{1 k} J_{15}^{(k)}, & i & =1, \\
K_{15} & =\sum_{k} \gamma_{5 k} J_{15}^{(k)}, & i & =5 .
\end{aligned}
$$


This independence of the index $i$ when matching first derivatives of the potential and actions is an alternate way to demonstrate the condition in (11) as manifested in (12) for the quadratic case.

Example 3 (Self-Interest Model of Action Functions). Consider the case where agents are acting out of self-interest only. Then the action function of agent $i$ will equal the quadratic payoff function (9) of agent $i$, and thus the gratitude configuration for utilities in (8) is

$$
\gamma_{i j}= \begin{cases}1 & i=j \\ 0 & i \neq j .\end{cases}
$$

Using the potential condition (12), the conditions for a potential in (14a), (14b), (14c), (14d) can be written as

$$
\begin{array}{cc}
K_{i j}=J_{i j}^{(i)}=J_{i j}^{(j)}, & i \leq j, \\
h_{i}=h_{i}^{(i)}, & 1 \leq i \leq N .
\end{array}
$$

The potential (13) is then

$$
V=\sum_{j \geq i=1}^{N} J_{i j}^{(i)} x_{i} x_{j}+\sum_{i=1}^{N} h_{i}^{(i)} x_{i}
$$

Example 4 (Quantity/Price-Payoff Model of Action Functions). The linear-demand Cournot model [5] as well as a two-market model of financial markets with speculators and hedgers $[8,9,10,11,7]$ have payoff functions of the form

$$
\pi_{k}(\mathbf{x})=x_{k}\left(\sum_{m \leq k} J_{m k}^{(k)} x_{m}+\sum_{m>k} J_{k m}^{(k)} x_{m}+h_{k}^{(k)}\right)+C_{k} .
$$

Note that in this model, $J_{i j}^{(k)}=0$ if $i \neq k$ or if $j \neq k$, and that $h_{j}^{(k)}=0$ if $j \neq k$. We wish to construct a quadratic potential (13) for the actions of the form (8) which are generated from the above payoff functions (19). The potential condition (12) can be reduced to

$$
\gamma_{i i} J_{i j}^{(i)}+\gamma_{i j} J_{i j}^{(j)}=\gamma_{j j} J_{i j}^{(j)}+\gamma_{j i} J_{i j}^{(i)}, \quad 1 \leq i<j \leq N .
$$

Since each agent has self-gratitude, $\gamma_{i i}=1$ for all $i$. To have a potential, it is therefore sufficient that the coefficients in the potential (13) and gratitude configuration satisfy

$$
\begin{array}{cc}
J_{i j}^{(i)}=J_{i j}^{(j)}, & 1 \leq i<j \leq N, \\
\gamma_{i j}=\gamma_{j i}, & 1 \leq i, j \leq N .
\end{array}
$$

Condition (21b) on the gratitude configuration is the case outlined in Example 2 of full agentexchange symmetry in the gratitude configuration.

Let

$$
\begin{aligned}
J_{i j} & :=J_{i j}^{(i)}=J_{i j}^{(j)}, \\
h_{i} & :=h_{i}^{(i)} .
\end{aligned}
$$


Using (14a), (14b), (14c), (14d), the potential (13) can be written

$$
V=\sum_{i=1}^{N} J_{i i} x_{i}^{2}+\sum_{i<j=1}^{N}\left(1+\gamma_{i j}\right) J_{i j} x_{i} x_{j}+\sum_{i=1}^{N} h_{i} x_{i}
$$

We will see subsequently that if bounded rationality is imposed on this example, and gratitude configurations are determined randomly and more slowly than equilibration, then the resulting model is a discrete-type of a "spin glass".

Example 4 is incomplete as presented above. It remains to be determined if it is an act of gratitude for one agent to add the payoff of another agent to their action function. Several examples below illustrate concepts in Humanomics that resolve this issue, along with Nash equilibrium analysis for the rational case (zero temperature) and correlation inequalities from statistical mechanics for the boundedly rational case (positive temperature).

Example 5 (Two Agents with Aligning Interactions). Consider a two-agent potential game, where agent 1 has strategy variable $x$ and agent 2 has strategy variable $y$. The payoffs

$$
\begin{aligned}
& \pi_{1}=x(-2 x+y+1), \\
& \pi_{2}=y(x-2 y+1),
\end{aligned}
$$

are aligning ('ferromagnetic' in physical systems) since the coefficients for the term xy are positive. The maximum of the corresponding potential

$$
V=-2 x^{2}-2 y^{2}+x y+x+y
$$

is a Nash equilibrium for the self-interest case where agents only maximize their own payoffs i.e., the actions equal the payoffs. This state of self-interest is reflected by a gratitude configuration (cf., Example 1) of

$$
\left(\gamma_{12}, \gamma_{21}\right)=(0,0), \quad \text { gratitude configuration for self-interest. }
$$

The self-interested action functions are then

$$
\begin{aligned}
& a_{1}=\pi_{1}=x(-2 x+y+1), \\
& a_{2}=\pi_{2}=y(x-2 y+1) .
\end{aligned}
$$

The self-interested Nash equilibrium occurs at $\left(x_{s}, y_{s}\right)=(1 / 3,1 / 3)$ and, using equation (25a) and equation (25b), corresponding payoffs

$$
\left(\pi_{1}^{s}, \pi_{2}^{s}\right)=(2 / 9,2 / 9)
$$

Now consider the one-way beneficence situation where agent 1 wants to show beneficence to agent 2. This gratitude configuration, which reflects this, is now

$$
\left(\gamma_{12}, \gamma_{21}\right)=(1,0), \quad \text { gratitude configuration for one-way beneficence of agent } 1 \text {. }
$$


Consequently, agent 1 takes action to reward agent 2 by adding the payoff of agent 2 to their own action. ${ }^{10}$ This results in the one-way beneficence action functions

$$
\begin{aligned}
& a_{1}=\pi_{1}+\pi_{2}=x(-2 x+y+1)+y(x-2 y+1), \\
& a_{2}=\pi_{2}=y(x-2 y+1) .
\end{aligned}
$$

The one-way beneficence Nash equilibrium occurs at $\left(x_{o}, y_{o}\right)=(3 / 7,5 / 14)$ with corresponding payoffs

$$
\left(\pi_{1}^{o}, \pi_{2}^{o}\right)=(3 / 14,25 / 98) .
$$

The intention of agent 1 is to make a gesture of beneficence towards agent 2. The result of the actions of agent 1 are that agent 1 receives less payoff than the self-interest case, and agent 2 receives more payoff than the self-interest case. A premise in Case 1 of the Desert Lemma in [1] (page 72) is satisfied, since agent 1 clearly did not have to help agent 2 gain more payoff (as is evident from agent 1 losing payoff as a result of this gesture). By Case 1 of the Desert Lemma, we will assume that agent 2 feels gratitude towards agent 1. From Axiom 3 [1] (page 71), gratitude most immediately and directly prompts agent 2 to reward agent 1.

As a result of the Desert Lemma and Axiom 3, we now have the case of mutual gratitude ${ }^{11}$ the gratitude configuration is now

$$
\left(\gamma_{12}, \gamma_{21}\right)=(1,1), \quad \text { gratitude configuration for mutual gratitude }
$$

The mutual-gratitude action functions are then

$$
\begin{aligned}
& a_{1}=\pi_{1}+\pi_{2}=x(-2 x+y+1)+y(x-2 y+1), \\
& a_{2}=\pi_{2}+\pi_{1}=y(x-2 y+1)+x(-2 x+y+1) .
\end{aligned}
$$

The mutual-gratitude Nash equilibrium occurs at $\left(x_{g}, y_{g}\right)=(1 / 2,1 / 2)$ with corresponding payoffs

$$
\left(\pi_{1}^{g}, \pi_{2}^{g}\right)=(1 / 4,1 / 4)
$$

Here we see that agent 2 reciprocates the beneficence back to agent 1, in accordance with Axiom 3 of [1]. The result is that agent 2 has lower payoff than the prior case (30). Agent 1 then has higher payoff than the prior case, and perceives that agent 2 helped them gain more payoff when agent 2 did not have to do so. By the previous argument, agent 1 will maintain a gratitude configuration of $\gamma_{12}=1$ and the condition in (33).

The end result is that both agents are better off showing mutual gratitude than self-interest. It is pointed out that this is not modeling collusion, but rather it is to be thought of as something

\footnotetext{
${ }^{10}$ We point out that the model assumes agents have learned that adding payoffs of other agents is an action that results in reward for this particular game. Agents initially would experiment with changes in action to determine if they result in reward or punishment. For example, if the agents were shop owners who sold widgets, then agent A could refer agent $\mathrm{B}$ to a widget producer $\mathrm{C}$ who is less expensive. If that producer gave terrible service, then the supposedly beneficent act was punishing instead or rewarding. Agent A could apologize to agent B to maintain mutual gratitude, and agent A would no longer refer other agents to producer $\mathrm{C}$.

${ }^{11}$ It may happen that agent 2 doesn't reward agent 1 . In this case it is possible, but not necessary, that agent 1 would revert back to no gratitude, which is the case in (27). The point is that people are complex and many choices are possible (c.f., [1] page 86 “... we cannot predict human conduct like we are able to predict water wave mechanics"), but we assume the more general case of reward to have a simplified but reasonable scenario in the spirit of this work.
} 
akin to agent 1 referring customers to agent 2, or agent 1 telling agent 2 about a beneficial discount on a good at a relatively unknown supplier. ${ }^{12}$

The process above can be reversed, and this remains faithful to the Desert Lemma and Axiom 3 of [1], by replacing the sentiment of gratitude with the sentiment of resentment. As such, it is reasonable for a simple model to assume mutual gratitude or mutual resentment as outlined in Example 2 for the case of games having the properties of this example.

Example 6 (Two Agents with Opposing Interactions). Consider a two-agent potential game, where agent 1 has strategy variable $x$ and agent 2 has strategy variable $y$. The payoffs

$$
\begin{aligned}
& \pi_{1}=x(-x-4 y+1), \\
& \pi_{2}=y(-4 x-y-1),
\end{aligned}
$$

are opposing ('antiferromagnetic' in physical systems) since the coefficients for the term xy are negative. We then have the following scenarios:

- self-interest where agents maximize their own payoffs - i.e., the actions equal the payoffs $a_{1}=\pi_{1}$ and $a_{2}=\pi_{2}$,

- one-way subtraction with actions $a_{1}^{-}=\pi_{1}-\pi_{2}$ and $a_{2}^{-}=\pi_{2}$,

- mutual subtraction with actions $a_{1}^{--}=\pi_{1}-\pi_{2}$ and $a_{2}^{--}=\pi_{2}-\pi_{1}$,

- one-way addition with actions $a_{1}^{+}=\pi_{1}+\pi_{2}$ and $a_{2}^{+}=\pi_{2}$,

- mutual addition with actions $a_{1}^{++}=a_{2}^{++}=\pi_{1}+\pi_{2}$.

The corresponding Nash equilibria and payoffs are summarized in Table 1.

\begin{tabular}{|c|c|c|c|}
\hline & case & Nash equilibrium $(x, y)$ & Payoffs $\left(\pi_{1}, \pi_{2}\right)$ \\
\hline 1 & self-interest & $(-1 / 2,1 / 2)$ & $(1 / 4,1 / 4)$ \\
\hline 2 & one-way subtraction & $(1 / 2,-3 / 2)$ & $(13 / 4,9 / 4)$ \\
\hline 3 & mutual subtraction & $(1 / 2,-1 / 2)$ & $(5 / 4,5 / 4)$ \\
\hline 4 & one-way addition & $(-5 / 14,3 / 14)$ & $(9 / 196,-5 / 28)$ \\
\hline 5 & mutual addition & $(-1 / 6,1 / 6)$ & $(-1 / 12,-1 / 12)$ \\
\hline
\end{tabular}

Table 1: Summary of Nash equilibria and payoffs for a case of opposing payoffs.

Unlike the aligning case in Example 5, we see in the "one-way addition" case ((4) of Table 1) that if agent 1 were to add the payoff of agent 2 to their action from the "self-interest" case (( 1$)$ of Table 1), the results would be decreased payoff for agent 2 as well as for agent 1 . Agent 1 did not have to add the payoff of agent 2 to their action, as is evident from the decrease in the payoff of agent 1. This premise appears in Case 2 of the Desert Lemma in [1], and by Case 2, agent 2 feels resentment towards agent 1 because of their decrease in payoff as a result of the actions of agent

\footnotetext{
${ }^{12}$ It is common experience that people will hesitate to tell others about a great deal, because as more people know about such a great offer, then less of the good at that discounted price will be available to the referring person. As a result they will have to buy more of the good at a higher price from a different source.
} 
1. That this decrease in payoff makes agent 2 feel worse off, and thus the application of Case 2, is a result of Axiom 0 of [1] (page 69) about human beings being non-satiated. Accordingly, Axiom 3 of [1] (page 71) prompts agent 2 to punish agent 1. Initially, agent 2 may not know enough about the game to know how to punish agent 1. But having seen that adding payoffs to actions resulted in punishment to agent 2, agent 2 may reciprocate this behavior and add the payoff of agent 1 to their action function. The result is the "mutual addition" case of (5) in Table 1, where indeed there is punishment towards agent 1 because of the decreased payoff of agent 1 along with Axiom 0 of [1].

In summary, we see that the game in this example has different characteristics than Example 5, where adding payoffs to actions is a sign of gratitude. Here the same action is a sign of resentment. This reinforces the ideas in Example 2 about agents needing knowledge about the intrinsics of the game in order to enact rewards or punishments through the alteration of action functions.

Example 7 (Many-Agents with Aligning Interactions). In a game where agents' strategy variables all have the same domain in some interval of real numbers, we can assume without loss of generality that the domain is centered at the origin (i.e., $\underline{x}=-|\bar{x}|=-\bar{x}$ in (3)) by transforming the coordinates as in [6,21]. Consider an N-agent game, with payoffs

$$
\pi_{i}=x_{i}\left(\frac{1}{N} \sum_{j \leq i} J_{j i} x_{j}+\frac{1}{N} \sum_{j>i} J_{i j} x_{j}+h_{i}\right),
$$

with $J_{i j} \geq 0$ (aligning interactions, or 'ferromagnetic' for physical problems) for $1 \leq i, j \leq N$ and $h_{i}, 1 \leq i \leq N$ all have the same sign. The inequality

$$
\begin{aligned}
\left|\pi_{i}\right| & =\left|\frac{J_{i i}}{N} x_{i}^{2}+x_{i}\left(h_{i}+\frac{1}{N} \sum_{j<i} J_{j i} x_{j}+\frac{1}{N} \sum_{j>i} J_{i j} x_{j}\right)\right| \\
& \leq \frac{J_{i i}}{N} x_{i}^{2}+\left|x_{i}\right|\left(\left|h_{i}\right|+\frac{1}{N} \sum_{j<i} J_{j i}\left|x_{j}\right|+\frac{1}{N} \sum_{j>i} J_{i j}\left|x_{j}\right|\right)
\end{aligned}
$$

shows that the maximum of the payoff will occur when all of the $x_{k}$ have the same sign as the $h_{i}$ and have maximum absolute value, in which case there will be equality in (39). If the $h_{i}$ are all zero, there will be two maxima: one at $x_{k}=\underline{x}$ for all $k$, and the other at $x_{k}=\bar{x}$ for all $k$. These type of 'aligning' payoffs occur in [9, 10, 11, 7] where speculators in a two-market system make money when they buy during positive excess demand and sell immediately after the purchase. ${ }^{13}$

In the self-interest case, each agent's action equals their own payoff. From the arguments above, we have the following Nash equilibrium(s):

$$
\begin{array}{lc}
\mathbf{x}^{\wedge}=(\underline{x}, \ldots, \underline{x}), & \text { all signs } h_{i}<0, \\
\mathbf{x}^{\wedge}=(\bar{x}, \ldots, \bar{x}), & \text { all signs } h_{i}>0, \\
\mathbf{x}_{ \pm}^{\wedge}= \pm(\bar{x}, \ldots, \bar{x}), & \text { all } h_{i}=0 .
\end{array}
$$

\footnotetext{
${ }^{13}$ The behavior from these types of interactions have been observed elsewhere in [24] as the 'hot-hand fallacy' in markets.
} 
In the case where each agent adds other agents' payoffs to their action function, as in (8), the action for agent $i$ satisfies the inequality

$$
\begin{aligned}
\left|a_{i}\right| & \leq \sum_{k=1}^{N} \gamma_{i k}\left|\pi_{k}\right| \\
& \leq \sum_{k=1}^{N} \gamma_{i k} \frac{J_{k k}}{N} x_{k}^{2}+\gamma_{i k}\left|x_{k}\left(h_{k}+\frac{1}{N} \sum_{j<k} J_{j k} x_{j}+\frac{1}{N} \sum_{j>k} J_{k j} x_{j}\right)\right|,
\end{aligned}
$$

where $\gamma_{i k}=0$ or $\gamma_{i k}=1$. As above, we see that each summand in (42) is maximized when all $x_{m}$ have the common sign of the $h_{k}$ and have maximum absolute value. If all $h_{k}=0$, there will be two maxima: one when all $x_{m}=\underline{x}$, and the other when all $x_{m}=\bar{x}$. In other words, the Nash equilibrium is the same as (40a), (40b), and (40c).

The key point of this example is that the Nash equilibrium does not change when agents add other agents' payoffs to their actions. As a result, the payoffs will be the same in the self-interest case and the case when agents add other agents' payoffs to their actions. Therefore,

$\star$ in this many-agent case with aligning interactions, adding payoffs is not a gesture of gratitude in the purely rational case (zero temperature).

\section{Bounded Rationality}

From Example 5 and Example 6, we see that an intrinsic knowledge of the game is necessary for agents to enact rewards or punishments. Initially, agents likely have no knowledge of what results will occur because of their actions. Indeed we saw that the addition of payoffs to actions can be a reward or a punishment. The more complex the game is, the more limited agents are in acquiring knowledge about the game. In [1] (page 15), it is mentioned that

... action is driven by discovery in a world of uncertainty and consequences that are unknown until attempted: through repeat social interactions and trade, people adapt their responses to better themselves as well as others through gains from exchange.

This is reiterated in [1] (page 55, paragraph 3):

... life is about "exploring" and "discovering" values rather than producing and enjoying them "to the greatest possible extent".

Finally, in [1] (page 59) it is mentioned that our senses are subject to error:

We can obviously sense a pattern in conduct, but it is a non-specifiable pattern subject to error.

To implement these ideas in a relatively simple manner, we investigate a dynamical approach of decision making based on localized decision making subject to random error (see also [25]). The stochastic dynamics, which are noisy perturbations of best-response dynamics that follow payoff gradients, are given by the Itô diffusion (Langevin) equations: for $1 \leq i \leq N$,

$$
d x_{i}=\frac{\partial}{\partial x_{i}} a_{i}(\mathbf{x}, t) d t+v d w_{i}(t)+r^{(i)}(\mathbf{x}) d z_{i}(t)
$$


where increments $w_{i}(t)-w_{i}(s)$ are zero-mean normal random variables with variance $t-s$ from a Wiener process (" $d w_{i}(t)$ ") is often referred to as a Gaussian white noise), $v$ is a variance parameter, $r^{(i)}(\mathbf{v})$ a one-dimensional reflection vector field, and $z_{i}(t)$ a process that only changes on the boundary for reflection. Here we use inward normal reflection on the boundary of the domain.

Using the definition of a potential (10), we can rewrite (43) above compactly as

$$
d \mathbf{x}=\nabla V d t+v d \mathbf{w}(t)+\mathbf{r}(\mathbf{x}) d \mathbf{z}(t)
$$

with $\mathbf{x}=\left(x_{1}, x_{2}, \ldots, x_{N}\right), d \mathbf{w}=\left(d w_{1}, d w_{2}, \ldots, d w_{N}\right), d \mathbf{z}=\left(d z_{1}, d z_{2}, \ldots, d z_{N}\right)$, and $\nabla V$ having components consistent with (43).

Below, we develop the stochastic dynamical system that yields the Gibbs measure as the stationary measure. See example 3.8 in [26] and previous arguments for a rigorous proof and also [27, 28, 29, 26, 30, 31] for stochastic/reflection and analytic techniques for semigroups and convergence rate bounds.

Proposition 1. Let $\rho(\mathbf{x})$ be the joint density function over decision space $\Phi_{\Lambda}$ for a potential game with a finite number of agents $N$ and potential $V$. Consider the dynamics ${ }^{14}$

$$
d \mathbf{x}=\nabla V d t+v d \mathbf{w}(t)+\mathbf{r}(\mathbf{x}) d \mathbf{z}(t),
$$

where $\mathbf{x} \in \Phi_{\Lambda}, \nabla V=\left(\partial V / \partial x_{1}, \partial V / \partial x_{2}, \ldots, \partial V / \partial x_{N}\right)$, and the $\mathbf{w}$ a vector of $N$ standard Wiener processes which are identical and independent across agents and time. Furthermore, the increments $w_{i}(t)-w_{i}(s)$, have mean zero, variance $t-s$, and reflecting boundary conditions ${ }^{15}$ are used. The matrix $\mathbf{r}$ is used for reflections at the boundary, and $\mathbf{z}(t)$ is a process that only changes on the boundary for reflection.

If the process $\mathbf{x}(t)$ satisfies the dynamics of (45), then the stationary density satisfies the Fokker-Planck equation

$$
\frac{\partial \rho(\mathbf{x}, t)}{\partial t}=0=-\nabla \cdot[\nabla V(\mathbf{x}(t)) \rho(\mathbf{x}, t)]+\frac{v^{2}}{2} \nabla^{2} \rho(\mathbf{x}, t)
$$

and hence the stationary measure for variance $v^{2}$ is the Gibbs state ([26] corollary 1 and example 3.8)

$$
\rho(\mathbf{x}, t)=\rho(\mathbf{x})=\frac{\exp \left(\frac{2}{v^{2}} V(\mathbf{x})\right)}{\int_{\Phi_{\Lambda}} \exp \left(\frac{2}{v^{2}} V\left(\mathbf{x}^{\prime}\right)\right) d \mathbf{x}^{\prime}} .
$$

where we use the convenient notation

$$
d \mathbf{x}=\prod_{i=1}^{N} d x_{i}
$$

In statistical mechanics, the term in the exponent of $(47)$ is $-E(\mathbf{x}) /(k T)$, where $k$ is Boltzmann's constant, $T$ is temperature, and $E(\mathbf{x})$ is the energy of configuration $\mathbf{x}$. Hence the analogy of a potential game to statistical mechanics is that $v^{2}$ (deviation from rationality; influence of

\footnotetext{
${ }^{14}$ Note that agents are only playing pure strategies in these dynamics.

${ }^{15}$ We use a normal reflection vector field on the boundary $\partial \Phi_{\Lambda}$ as in [26].
} 
the noise in dynamics (43)) is proportional to 'temperature' and the potential $V$ is the negative 'energy' of the system [6].

An alternate approach that yields the Gibbs measure is a maximum information-entropy solution where the potential is constrained to have a fixed mean (see [6]). If the potential is constrained to have a mean below its maximum, then this constraint is enforcing the degree of bounded rationality. This is because perfectly rational agents would choose the maximum of the potential. In the constrained maximum entropy view, where the mean potential is constrained, agents play other strategies and "arbitrage" information out of the system by exploring other strategies to try to improve payoff.

A fluctuation-dissipation argument then relates the constrained maximum information entropy solution to the stochastic dynamical solution (both Gibbs measures) and the "temperature" $T$ is seen to be proportional to the square of the fluctuation parameter $v^{2}$ by

$$
T:=1 / \beta=\frac{v^{2}}{2} \text {. }
$$

This results in the measure

$$
\rho(\mathbf{x}):=\frac{\exp (\beta V(\mathbf{x}))}{\int_{\Phi_{\Lambda}} \exp \left(\beta V\left(\mathbf{x}^{\prime}\right)\right) d \mathbf{x}^{\prime}},
$$

Finally, we note that maximums of the potential are the appropriate refinement of the Nash equilibriums for a potential game (cf., [32]).

An observable $g \in C\left(\Phi_{\Lambda}\right)$ in the economic system is a continuous function on pure state space of strategy variables such as a payoff $\pi_{i}(\mathbf{x})$, inverse demand $p(\mathbf{x})$, quantity purchased by a particular agent $x_{k}$, etc. We denote the expected value of an observable $g$ with respect to the Gibbs state (50) by

$$
\langle g\rangle:=\int_{\Phi_{\Lambda}} g(\mathbf{x}) \rho(\mathbf{x}) d \mathbf{x} .
$$

\section{Beneficence in a Boundedly Rational Case of Many-Agents with Aligning Interactions}

We saw in Example 7 that agents do not benefit when their payoff is added to another agent's action function. That scenario was for the rational case, when agents have complete knowledge of the economic system. In [21] a similar result was established, and it was also seen that bounded rationality limited the agents knowledge of the system and that mutual "coopetition" (cooperative competition; e.g., [33]) resulting in agents adding other agents' payoffs to their coopetitive payoff functions resulted in increased payoffs for all agents. Thus agents working together in this coopetitive manner has the effect of increasing rationality, in the sense that agents can produce higher payoffs for themselves via coopetition than they can in the self-interest case of working alone.

Coopetition can mitigate the effects of incomplete information about the economic system for that case of aligning interactions. Since the interactions in Example 7 are aligning, there is good reason to suspect that same result holds here for boundedly rational agents. That result, in this context of beneficence, would imply that adding payoffs to actions is a sign of beneficence. To that end, the boundedly rational scenario of Example 7 with the potential conditions of Example 4 is obtained by using the payoffs in (37), along with a gratitude configuration $\gamma$, to obtain actions

$$
a_{i}=\sum_{k=1}^{N} \gamma_{i k} \frac{J_{k k}}{N} x_{k}^{2}+\gamma_{i k}\left[x_{k}\left(\frac{1}{N} \sum_{j<k} J_{j k} x_{j}+\frac{1}{N} \sum_{j>k} J_{k j} x_{j}+h_{k}\right)\right],
$$


where for all $1 \leq i, j \leq N$,

$$
\begin{array}{r}
J_{i j} \geq 0 \\
\operatorname{sign}\left(h_{i}\right)=\operatorname{sign}\left(h_{j}\right) \\
\gamma_{i j}=\gamma_{j i} \in\{0,1\} \\
x_{i} \in[\underline{x}, \bar{x}] .
\end{array}
$$

(all $h_{i}$ terms have the same sign), (53b)

(reciprocation of resentment/gratitude), (53c)

A potential for the actions in (52), as in (24), is

$$
V(\mathbf{x} ; \gamma)=\frac{1}{N} \sum_{i=1}^{N} J_{i i} x_{i}^{2}+\frac{1}{N} \sum_{i<j=1}^{N}\left(1+\gamma_{i j}\right) J_{i j} x_{i} x_{j}+\sum_{i=1}^{N} h_{i} x_{i} .
$$

\subsection{Slow Gratitude Alterations and Spin Glass Models}

One question not yet raised is the time scale of the alteration of gratitude interactions versus the time scale of economic equilibrium. Here we look at the case when gratitude configurations form significantly more slowly that the time needed for economic equilibrium. An example of this case would be the fast trading speculators in the model studied in $[8,9,10,11,7]$. Since "high frequency' traders often arbitrage inefficiencies out of the market in the order of milliseconds, any gratitude or resentment that a trader has for another trader over that time period will remain relatively constant. Thus economic equilibrium will occur while the gratitude configuration $\gamma$ is constant. Expectations can then be taken over the probability distribution function of the gratitude variables to obtain mean values.

From Section 5, the equilibrium measure for the boundedly rational scenario of the noisydecision dynamics of (44) is obtained from (51) as

$$
\langle g\rangle_{\gamma}:=\int_{\Phi_{\Lambda}} g(\mathbf{x}) \rho_{\gamma}(\mathbf{x}) d \mathbf{x}
$$

for any observable $g \in C\left(\Phi_{\Lambda}\right)$, where $\gamma$ specifies a gratitude configuration and

$$
\rho_{\gamma}(\mathbf{x})=\frac{\exp [\beta V(\mathbf{x} ; \gamma)]}{\int_{\Phi_{\Lambda}} \exp \left[\beta V\left(\mathbf{x}^{\prime} ; \gamma\right)\right] d \mathbf{x}^{\prime}} .
$$

The partition function is defined as

$$
\mathcal{Z}_{\gamma}=\mathcal{Z}_{\gamma}\left(\beta,\left\{J_{i j}\right\},\left\{h_{i}\right\},\left\{\gamma_{i j}\right\} ; N\right):=\int_{\Phi_{\Lambda}} \exp [\beta V(\mathbf{x} ; \gamma)] d \mathbf{x} .
$$

We regard the gratitude configuration variables an continuous in the interval $[0,1]$ to determine the change in mean payoff with respect to the gratitude variables $\gamma_{i j}$, which is

$$
\begin{aligned}
\frac{\partial}{\partial \gamma_{m n}}\left\langle\pi_{i}\right\rangle_{\gamma} & =\frac{\partial}{\partial \gamma_{m n}} \frac{\int_{\Phi_{\Lambda}} \exp [\beta V(\mathbf{x} ; \gamma)] \pi_{i}(\mathbf{x}) d \mathbf{x}}{\mathcal{Z}_{\gamma}} \\
& =\beta\left\langle\pi_{i} \frac{\partial V}{\partial \gamma_{m n}}\right\rangle_{\gamma}-\beta\left\langle\pi_{i}\right\rangle_{\gamma}\left\langle\frac{\partial V}{\partial \gamma_{m n}}\right\rangle_{\gamma},
\end{aligned}
$$


which is a correlation function. Since we are considering the case specified in (53b), if the signs of all $h_{i}$ are negative in (54), we can change variables from $x_{i}$ to $-x_{i}$ for all $i$ in (56) and (57) and effectively change the sign of all $h_{i}$ to be nonnegative. Therefore, without loss of generality, we assume the sign of each $h_{i}$ in (54) is nonnegative. Since each $J_{i j}$ is nonnegative in (13), the correlation in (59) is positive by using the techniques in [21]. We outline this and a more general technique to prove positive correlations in Appendix A which may be helpful if agents were to add functions other than payoffs to actions, or if the payoffs are increasing but do not have all positive coefficients.

The addition of payoffs is thus a sign of gratitude when averaged within a boundedly rational economic equilibrium using the Gibbs measure. This is in contrast to the perfectly rational case, i.e. Nash equilibrium, where the addition of payoffs to actions would not be regarded as a gesture of gratitude as outlined in Example 7.

Finally, we see that long-run values of observables are obtained by taking the gratitude expectation of the expected value of the observable with respect to the Gibbs measure; i.e.,

$$
\mathbb{E}_{\gamma}\left[\langle g\rangle_{\gamma}\right],
$$

where $\mathbb{E}_{\gamma}$ is the expectation with respect to the gratitude variables, and $g \in C\left(\Phi_{\Lambda}\right)$ is an observable. A specific case of this is done by taking derivatives of the prequenched free potential,

$$
f_{\gamma, N}=\frac{1}{\beta N} \ln \left(\mathcal{Z}_{\gamma}\right)
$$

and then averaging over gratitude variables, which is equivalent to taking the same derivatives of the quenched free potential

$$
\bar{f}_{N}=\frac{1}{\beta N} \mathbb{E}_{\gamma}\left[\ln \left(\mathcal{Z}_{\gamma}\right)\right] .
$$

From the above, we see that slow gratitude alterations result in a 'spin glass' model. We point out that positive correlations of payoff functions require the interactions to be nonnegative:

$$
\left(1+\gamma_{i j}\right) J_{i j} \geq 0,
$$

for all $i, j \in \Lambda$. This constrains the gratitude configuration variables to $\gamma_{i j} \geq-1$. When these random gratitude variables have minimum value, the effect is disorder (an interaction closer to zero is effectively higher temperature - less correlation and more independent random behavior). However, this disorder is a result of less correlation, or paramagnetic/thermal, rather than a result of antiferromagnetic behavior resulting from negative interactions in a traditional spin glass.

\subsection{Fast Gratitude Alterations and Annealed Models}

Consider the case when gratitude configurations change significantly faster that the time needed for economic equilibrium. An example of this case would be the long-term hedging agent in the model studied in $[8,9,10,11,7]$. Such 'slow' traders buy enough product to last the order of 6 months or more (for example, someone investing in their retirement is looking at 30 years or more), over which time significant interactions can occur that result in gratitude and resentment. Thus, economic equilibrium will occur while the gratitude configuration $\gamma$ changes. A first-order approximation to economic equilibrium would be to have the economic dynamics taken over expected values of the gratitude variables to obtain mean economic-dynamical behavior of agents. 
To that end, we consider the mean of the potential in (54) over gratitude variables. Denote the mean value of a gratitude variable by

$$
\overline{\gamma_{i j}}:=\mathbb{E}_{\gamma}\left[\gamma_{i j}\right] .
$$

The boundedly rational dynamics (45) then uses the mean of the potential

$$
\bar{V}(\mathbf{x})=\mathbb{E}_{\gamma}[V(\mathbf{x} ; \gamma)]=\frac{1}{N} \sum_{i=1}^{N} J_{i i} x_{i}^{2}+\frac{1}{N} \sum_{i<j=1}^{N}\left(1+\overline{\gamma_{i j}}\right) J_{i j} x_{i} x_{j}+\sum_{i=1}^{N} h_{i} x_{i} .
$$

The equilibrium is then the Gibbs measure (50) with linear coefficients $h_{i}$ and constant quadratic coefficients $J_{i i}$ and $\left(1+\overline{\gamma_{i j}}\right) J_{i j}$ for $i<j$.

\section{Two-Person Aligning Game}

Here we will model a basic two-person non-trivial quadratic model with an aligning (ferromagnetic) interaction. In this model, we assume that economic equilibrium occurs faster than the changing of gratitude and resentment, such as with high-frequency traders who trade very quickly (e.g. the speculators in [9]). As such, this is a quenched model, and the gratitude configuration is constant during the formation of economic equilibrium at any specific time. Gratitude configurations then form at an adequately slower rate than economic equilibrium.

We consider an interaction-heterogeneous scenario, where ex ante heterogeneity is modeled by different linear terms as in [12, section 3.4.3]. Such heterogeneity allows for model data fitting and testing, and can represent differences among agents in characteristics like age, race, gender, and education, to name a few. Here we will see that the heterogeneity represents different levels of payoff. In [1] a majority of the experimental choices we report in Chapters 10 and 13 (13 is more measurement than prediction) are predicted by Adam Smith's propositions summarizing the predictive consequences of his model of human sociability. A minority fail; moreover there is evidence that some of these failures (and maybe some of the successes-errors go both ways!) are consequences of people mis-reading the meaning signalled in the actions. In other words we have no shortage of agent heterogeneity. The propositions in TMS [2] are derived from assumptions that allow one to predict what might have caused contra-predicted actions; e.g., no feelings of gratitude; or feelings not strong enough to overcome the payoff from defection (test by varying payoff from defection and if they make no difference it suggests no feelings of gratitude-sociopathology), and so on. This is a plus for TMS [2] for it has something positive to say about actor heterogeneity.

Agent 1 has strategy variable $x_{1} \in[\underline{x}, \bar{x}]$ and agent 2 has strategy variable $x_{2} \in[\underline{x}, \bar{x}]$, with $\bar{x}=-\underline{x}>0$. The payoffs for agent 1 and agent 2 are

$$
\begin{aligned}
& \pi_{1}=x_{1}\left(J x_{2}+h_{1}\right), \\
& \pi_{2}=x_{2}\left(J x_{1}+h_{2}\right),
\end{aligned}
$$

where $J>0, h_{1}>0$, and $h_{2}>0$.

The (mutual) gratitude configuration is a single random variable $\gamma=\gamma_{1,2} \in\left\{w_{g}, w_{r}\right\},-1 \leq$ $w_{r}<w_{g}$, where $\gamma=w_{g}$ indicates mutual gratitude and $\gamma=w_{r}$ indicates mutual resentment, since 
mean payoffs (with respect to the Gibbs measure) are increasing in $\gamma$. We assume a quenched probability distribution function $\Phi$ for the random variable $\gamma$, with

$$
\Phi(\gamma)= \begin{cases}p & \gamma=w_{g}(\text { gratitude }) \\ 1-p & \gamma=w_{r}(\text { resentment })\end{cases}
$$

where $0 \leq p \leq 1$.

The actions for the agents are

$$
\begin{aligned}
& a_{1}=\pi_{1}+\gamma \pi_{2}=(1+\gamma) J x_{1} x_{2}+h_{1} x_{1}+\gamma h_{2} x_{2}, \\
& a_{2}=\pi_{2}+\gamma \pi_{1}=(1+\gamma) J x_{1} x_{2}+h_{2} x_{2}+\gamma h_{1} x_{1},
\end{aligned}
$$

and a potential for this model is

$$
V(\mathbf{x} ; \gamma)=(1+\gamma) J x_{1} x_{2}+h_{1} x_{1}+h_{2} x_{2} .
$$

With bounded rationality parameter $\beta$ (inverse temperature), the boundedly rational equilibrium is a Gibbs measure with partition function

$$
\begin{aligned}
\mathcal{Z}_{\gamma} & =\int \exp [\beta V(\mathbf{x} ; \gamma)] d \mathbf{x} \\
& =\int_{\underline{x}}^{\bar{x}} \int_{\underline{x}}^{\bar{x}} \exp \left[\beta(1+\gamma) J x_{1} x_{2}+\beta h_{1} x_{1}+\beta h_{2} x_{2}\right] d x_{1} d x_{2} .
\end{aligned}
$$

If $\gamma=-1$, then the integral is

$$
\begin{aligned}
\mathcal{Z}_{\gamma=-1} & =\int_{\underline{x}}^{\bar{x}} \int_{\underline{x}}^{\bar{x}} \exp \left[\beta h_{1} x_{1}+\beta h_{2} x_{2}\right] d x_{1} d x_{2} \\
& =\frac{4}{\beta^{2} h_{1} h_{2}} \sinh \left(\beta h_{1} \bar{x}\right) \sinh \left(\beta h_{2} \bar{x}\right) .
\end{aligned}
$$

Otherwise, if $\gamma \neq-1$, integrating with respect to $x_{2}$ yields

$$
\mathcal{Z}_{\gamma}=\int_{\underline{x}}^{\bar{x}} \exp \left[\beta h_{1} x_{1}\right] \frac{2 \sinh \left(\beta\left[(1+\gamma) J x_{1}+h_{2}\right] \bar{x}\right)}{\beta\left[(1+\gamma) J x_{1}+h_{2}\right]} d x_{1} .
$$

Note that integrand in (74) is integrable in $x_{1}$. With the substitutions

$$
\begin{aligned}
u & =\beta\left[(1+\gamma) J x_{1}+h_{2}\right], \\
\alpha_{\gamma} & :=\frac{h_{1}}{(1+\gamma) J},
\end{aligned}
$$

the integral (74) can be rewritten as

$$
\mathcal{Z}_{\gamma}=\frac{\exp \left(-\beta h_{2} \alpha_{\gamma}\right)}{\beta(1+\gamma) J} \int_{\underline{u}}^{\bar{u}}\left[\frac{e^{(\alpha+\bar{x}) u}}{u}-\frac{e^{(\alpha-\bar{x}) u}}{u}\right] d u .
$$

To accommodate analytic results, we use the functions

$$
\begin{aligned}
& \operatorname{Ei}(x):=\int_{-\infty}^{x} \frac{e^{t}}{t} d t, \quad x<0, \\
& \operatorname{Shi}(x):=\int_{0}^{x} \frac{\sinh (t)}{t} d t, \quad x \in(-\infty, \infty) .
\end{aligned}
$$


Using principal values, $\operatorname{Ei}(x)$ can be extended to $x>0$ in addition. For $x>0$ this is equivalent to the formula

$$
\operatorname{Ei}(x):=\operatorname{Ei}(-x)+2 \operatorname{Shi}(x), \quad x>0 .
$$

Provided that $\alpha_{\gamma} \pm \underline{x}$ and $\alpha_{\gamma} \pm \bar{x}$ are nonzero, the integral in (77) is then

$$
\mathcal{Z}_{\gamma}=\frac{\exp \left(-\beta h_{2} \alpha_{\gamma}\right)}{\beta(1+\gamma) J}\left[\left\{\operatorname{Ei}\left(\left[\alpha_{\gamma}+\bar{x}\right] \bar{u}\right)-\operatorname{Ei}\left(\left[\alpha_{\gamma}-\bar{x}\right] \bar{u}\right)\right\}-\left\{\operatorname{Ei}\left(\left[\alpha_{\gamma}+\bar{x}\right] \underline{u}\right)-\operatorname{Ei}\left(\left[\alpha_{\gamma}-\bar{x}\right] \underline{u}\right)\right\}\right],
$$

where if either $\bar{u}=0$ or $\underline{u}=0$, we use the principal value limits and the boundedness of $\exp (x)$ on $\left[\left(\alpha_{\gamma}-\bar{x}\right) \epsilon,\left(\alpha_{\gamma}+\bar{x}\right) \epsilon\right]$ as $\epsilon \rightarrow 0$ to obtain

$$
\lim _{u \rightarrow 0} \operatorname{Ei}\left(\left[\alpha_{\gamma}+\bar{x}\right] u\right)-\operatorname{Ei}\left(\left[\alpha_{\gamma}-\bar{x}\right] u\right)=\ln \frac{\left|\alpha_{\gamma}+\bar{x}\right|}{\left|\alpha_{\gamma}-\bar{x}\right|}
$$

when $\alpha_{\gamma}+\bar{x}$ and $\alpha_{\gamma}-\bar{x}$ have the same sign. When $\alpha_{\gamma}+\bar{x}>0$ and $\alpha_{\gamma}-\bar{x}<0$ we use

$$
\begin{aligned}
\lim _{u \rightarrow 0} \operatorname{Ei}\left(\left[\alpha_{\gamma}+\bar{x}\right] u\right)-\operatorname{Ei}\left(\left[\alpha_{\gamma}-\bar{x}\right] u\right) & =\lim _{u \rightarrow 0} \operatorname{Ei}\left(\left[\alpha_{\gamma}+\bar{x}\right] u\right)-\operatorname{Ei}\left(-\left[\alpha_{\gamma}-\bar{x}\right] u\right)+2 \operatorname{Shi}\left(-\left[\alpha_{\gamma}-\bar{x}\right] u\right) \\
& =\ln \frac{\alpha_{\gamma}+\bar{x}}{\left|\alpha_{\gamma}-\bar{x}\right|} .
\end{aligned}
$$

In all cases when $\alpha_{\gamma}+\underline{x}$ and $\alpha_{\gamma}-\underline{x}$ are nonzero, the partition function can be evaluated analytically using (80) with the zero limits

$$
\operatorname{Ei}\left(\left[\alpha_{\gamma}+\bar{x}\right] 0^{+}\right)-\operatorname{Ei}\left(\left[\alpha_{\gamma}-\bar{x}\right] 0^{+}\right)=\ln \frac{\left|\alpha_{\gamma}+\bar{x}\right|}{\left|\alpha_{\gamma}-\bar{x}\right|} .
$$

If $\alpha_{\gamma}+\underline{x}=0$ or $\alpha_{\gamma}-\underline{x}=0$, evaluating the partition function amounts to evaluating an integral of the form

$$
\int_{\underline{u}}^{\bar{u}} \frac{e^{u}-1}{u} d u .
$$

The integrand in (84) is increasing, with a finite limit at negative infinity, and is thus integrable on any finite interval. Furthermore, the integral can be evaluated using the functions $\operatorname{Ei}(x)$ and $\ln (x)$ with appropriate limits taken as above.

\subsection{Computational Results for Two-Person Quenched Game}

Here we show the computations for expected values of payoffs with respect to the Gibbs measure as well as their quenched means for the cases of parameters that yield the partition function in (80):

$$
\begin{aligned}
\left(\alpha_{\gamma} \pm \underline{x}\right) & \neq 0, \\
\left(\alpha_{\gamma} \pm \bar{x}\right) & \neq 0, \\
\underline{u} & \neq 0, \\
\bar{u} & \neq 0,
\end{aligned}
$$

where the mutual resentment case for the partition function, $\gamma=-1$, is given by (73). Other cases can be computed in a similar manner as below. 
Using (73), the expected values for $\gamma=-1$ of the payoffs (66) and (67) are

$$
\begin{aligned}
& \left\langle\pi_{1}\right\rangle_{\gamma=-1}=\left(\bar{x} \operatorname{coth}\left(\beta h_{1} \bar{x}\right)-\frac{1}{\beta h_{1}}\right)\left[J\left(\bar{x} \operatorname{coth}\left(\beta h_{2} \bar{x}\right)-\frac{1}{\beta h_{2}}\right)+h_{1}\right], \\
& \left\langle\pi_{2}\right\rangle_{\gamma=-1}=\left(\bar{x} \operatorname{coth}\left(\beta h_{2} \bar{x}\right)-\frac{1}{\beta h_{2}}\right)\left[J\left(\bar{x} \operatorname{coth}\left(\beta h_{1} \bar{x}\right)-\frac{1}{\beta h_{1}}\right)+h_{2}\right] .
\end{aligned}
$$

Similarly, using (80), the expected values for $\gamma>-1$ of the payoffs (66) and (67) are found from three expected values. The first is

$$
\begin{aligned}
& \left\langle x_{1} x_{2}\right\rangle_{\gamma>-1}=\left.\frac{1}{\beta(1+\gamma)} \frac{\partial}{\partial J}\right|_{\gamma>-1} \ln \mathcal{Z}_{\gamma}
\end{aligned}
$$

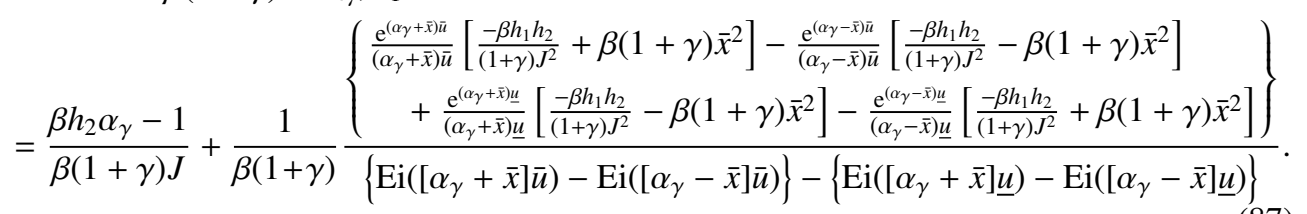

Likewise,

$$
\begin{aligned}
& \left\langle x_{1}\right\rangle_{\gamma>-1}=\left.\frac{1}{\beta} \frac{\partial}{\partial h_{1}}\right|_{\gamma>-1} \ln \mathcal{Z}_{\gamma} \\
& =\frac{-h_{2}}{(1+\gamma) J}+\frac{1}{\beta} \frac{\left\{\begin{array}{c}
\frac{\mathrm{e}^{(\alpha \gamma+\bar{x}) \bar{u}}}{\left(\alpha_{\gamma}+\bar{x}\right) \bar{u}}\left[\frac{\beta h_{2}}{(1+\gamma) J}+\beta \bar{x}\right]-\frac{\mathrm{e}^{(\alpha \gamma-\bar{x}) \bar{u}}}{\left(\alpha_{\gamma}-\bar{x}\right) \bar{u}}\left[\frac{\beta h_{2}}{(1+\gamma) J}+\beta \bar{x}\right] \\
\left.+\frac{\mathrm{e}^{(\alpha \gamma+\bar{x})} \underline{u}}{\left(\alpha_{\gamma}+\bar{x}\right) \underline{u}}\left[\frac{\beta h_{2}}{(1+\gamma) J}-\beta \bar{x}\right]-\frac{\mathrm{e}^{(\alpha \gamma-\bar{x}) \underline{u}}\left(\alpha_{\gamma}-\bar{x}\right) \underline{u}}{\left(\frac{\beta h_{2}}{(1+\gamma) J}-\beta \bar{x}\right]}\right\}
\end{array}\right\}}{\left.\operatorname{Ei}\left(\left[\alpha_{\gamma}+\bar{x}\right] \bar{u}\right)-\operatorname{Ei}\left(\left[\alpha_{\gamma}-\bar{x}\right] \bar{u}\right)\right\}-\left\{\operatorname{Ei}\left(\left[\alpha_{\gamma}+\bar{x}\right] \underline{u}\right)-\operatorname{Ei}\left(\left[\alpha_{\gamma}-\bar{x}\right] \underline{u}\right)\right\}}
\end{aligned}
$$

and

$$
\begin{aligned}
& \left\langle x_{2}\right\rangle_{\gamma>-1}=\left.\frac{1}{\beta} \frac{\partial}{\partial h_{2}}\right|_{\gamma>-1} \ln \mathcal{Z}_{\gamma} \\
& =\frac{-h_{1}}{(1+\gamma) J}+\frac{1}{\beta} \frac{\left\{\begin{array}{c}
\frac{\mathrm{e}^{\left(\alpha_{\gamma}+\bar{x}\right) \bar{u}}}{\left(\alpha_{\gamma}+\bar{x}\right) \bar{u}}\left[\frac{\beta h_{1}}{(1+\gamma) J}+\beta \bar{x}\right]-\frac{\mathrm{e}^{\left(\alpha_{\gamma}-\bar{x}\right) \bar{u}}}{\left(\alpha_{\gamma}-\bar{x}\right) \bar{u}}\left[\frac{\beta h_{1}}{(1+\gamma) J}-\beta \bar{x}\right] \\
+\frac{\mathrm{e}^{\left(\alpha_{\gamma}+\bar{x}\right) \underline{u}}}{\left(\alpha_{\gamma}+\bar{x}\right) \underline{u}}\left[\frac{\beta h_{1}}{(1+\gamma) J}+\beta \bar{x}\right]-\frac{\mathrm{e}^{\left(\alpha_{\gamma}-\bar{x}\right) \underline{u}}}{\left(\alpha_{\gamma}-\bar{x}\right) \underline{u}}\left[\frac{\beta h_{1}}{(1+\gamma) J}-\beta \bar{x}\right]
\end{array}\right\}}{\left.\operatorname{Ei}\left(\left[\alpha_{\gamma}+\bar{x}\right] \bar{u}\right)-\operatorname{Ei}\left(\left[\alpha_{\gamma}-\bar{x}\right] \bar{u}\right)\right\}-\left\{\operatorname{Ei}\left(\left[\alpha_{\gamma}+\bar{x}\right] \underline{u}\right)-\operatorname{Ei}\left(\left[\alpha_{\gamma}-\bar{x}\right] \underline{u}\right)\right\}}
\end{aligned}
$$

The expected gratitude payoffs with respect to the Gibbs measure can then be computed using (66) and (67) with (87), (88), and (89) to be

$$
\begin{aligned}
& \left\langle\pi_{1}\right\rangle_{\gamma>-1}=J\left\langle x_{1} x_{2}\right\rangle_{\gamma>-1}+h_{1}\left\langle x_{1}\right\rangle_{\gamma>-1}, \\
& \left\langle\pi_{2}\right\rangle_{\gamma>-1}=J\left\langle x_{1} x_{2}\right\rangle_{\gamma>-1}+h_{2}\left\langle x_{2}\right\rangle_{\gamma>-1} .
\end{aligned}
$$

Finally, we take quenched averages over $\Phi$ to get expected values of payoffs that consider the effects of mutual gratitude and resentment between the agents:

$$
\begin{aligned}
& \overline{\pi_{1}}:=\mathbb{E}_{\gamma}\left[\left\langle\pi_{1}\right\rangle_{\gamma}\right]=\left\langle\pi_{1}\right\rangle_{\gamma=w_{r}} \Phi\left(w_{r}\right)+\left\langle\pi_{1}\right\rangle_{\gamma=w_{g}} \Phi\left(w_{g}\right), \\
& \overline{\pi_{2}}:=\mathbb{E}_{\gamma}\left[\left\langle\pi_{2}\right\rangle_{\gamma}\right]=\left\langle\pi_{2}\right\rangle_{\gamma=w_{r}} \Phi\left(w_{r}\right)+\left\langle\pi_{2}\right\rangle_{\gamma=w_{g}} \Phi\left(w_{g}\right),
\end{aligned}
$$

where the formulas from (66), (67), (90a), and (90b) are used for the computation. 


\subsubsection{Numerical Results for Two-Person Quenched Game}

The results for the model presented in Section 7.1 for the parameter values $J=4, h_{1}=1$, $h_{2}=6, \bar{x}=1$, mutual resentment weight $\gamma=\gamma_{12}=w_{r}=-1$, and mutual gratitude weight $\gamma=\gamma_{12}=w_{g}=1$ are shown in Figure 1 .

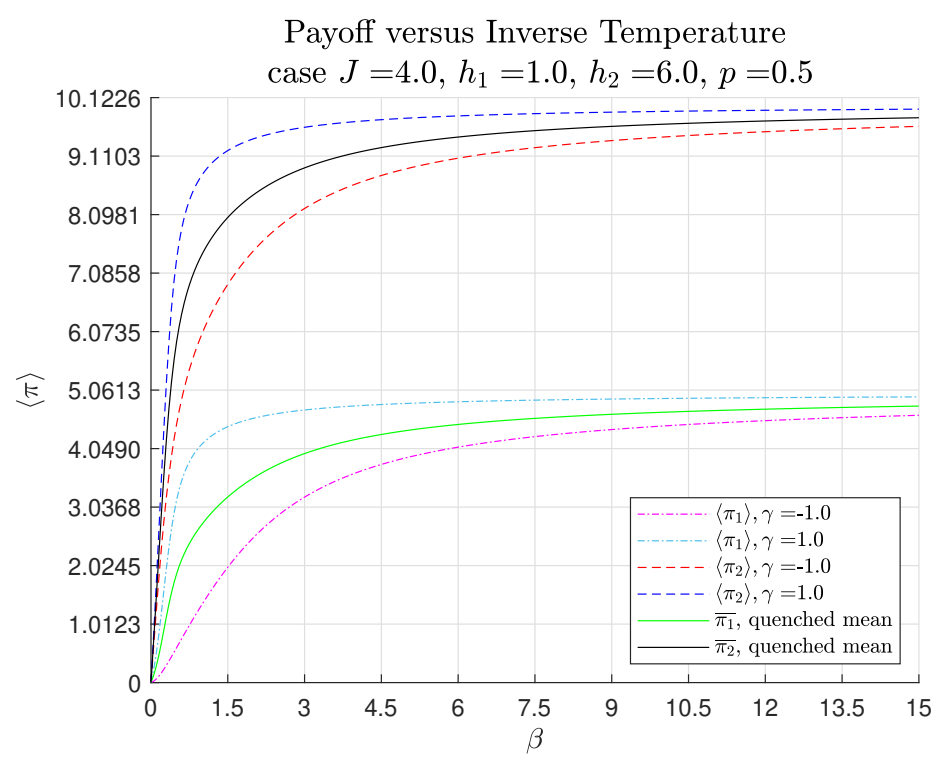

Figure 1: Two-person quenched game with quench probability of mutual gratitude, $p=0.5$, and mutual resentment $1-p=0.5$. At $\beta=0$, behavior is purely random (Gibbs measure is uniform), hence all payoffs are zero from (90a) and (90b). As $\beta$ approaches infinity, the payoffs approach the Nash equilibrium, and the gratitude and resentment payoffs are equal there by the arguments in Example 7. It is clear that mutual gratitude results in higher payoffs than mutual resentment, and that mean payoffs are increasing in $\beta$ as a result of correlation inequalities in Appendix A.

We see in Figure 1 that as agents become more non-rational, i.e. $\beta \rightarrow 0$, the difference in payoff in the gratitude state, $\gamma=1$, and resentment state, $\gamma=-1$, goes to zero. This is because agent decisions become more random, and at $\beta=0$ agent decisions are purely random in a uniform distribution. If people behave randomly, then fundamental ideas like universal nonsatiability do not hold. Therefore it is impossible for one agent to know what is good or bad for another agent, and thus impossible for an agent to reward or punish another agent. The $\beta=0$ result will hold for all boundedly rational potential games that have the Gibbs measure (56) as stationary measure for equilibrium.

It is also evident that the difference in payoff in the gratitude state and resentment state goes to zero in the purely rational limit $\beta \rightarrow \infty$. It was shown in Example 7 that for this particular game, agents can find a state where the difference in payoff between the gratitude state and resentment state is zero in the perfectly rational case. In other words, agents can choose a strategy which makes the expression of gratitude (the addition of another's payoff to one's action function) irrelevant to mean payoff. In this case, the rules of this particular game limit the effects of gratitude for perfectly rational agents when they play the optimal strategy.

In [1] (end of chapter 11) it is mentioned that people learn from their behavior, and as a result 
moderate their behavior to achieve societal reward for said behavior. The example used in [1] is of a child who receives indulgence from parents at a young age, but no longer receives such indulgence from peers once starting school. We capture some of that process in this example with bounded rationality. We can perceive the child to be acting on partial information before starting school, with a high degree of non-rationality ( $\operatorname{cmall} \beta \approx 0$ in Figure 1 where the difference in payoff for gratitude and resentment states is small). When the child goes to school and gains more information about how peers act, the child becomes less non-rational and differences in "payoff" between resentment and gratitude states becomes larger (as in Figure 1 for $\beta \approx 1.5$ ). As such, the child is more inclined to behave in a way that is conducive to mutual gratitude and to avoid mutual resentment.

\subsection{Critical Quenched Probability to Overcome Self-Interested Behavior}

Agents may behave in a self-interested manner, for which the gratitude configuration is $\gamma=0$ in the potential (71). This will clearly have higher payoff to all agents than if the quenched model has all weight on the resentment configuration, $\gamma=-1$, i.e., $p=0$ in (68). This is because the mean payoffs in (90a) and (90b) are strictly increasing in $\gamma$, which is evident from (59) and the comments below it. Since

$$
\left\langle\pi_{i}\right\rangle_{\gamma=1}>\left\langle\pi_{i}\right\rangle_{\gamma=-1}
$$

for $i=1,2$, the quenched payoffs

$$
\overline{\pi_{i}}:=\mathbb{E}_{\gamma}\left[\left\langle\pi_{i}\right\rangle_{\gamma}\right]=\left\langle\pi_{i}\right\rangle_{\gamma=-1}(1-p)+\left\langle\pi_{i}\right\rangle_{\gamma=1} p,
$$

$i=1,2$, are strictly increasing in $p$, the quenched weight on mutual gratitude which represents the ratio of time that agents have mutual gratitude for each other. It follows from the inequalities

$$
\left\langle\pi_{i}\right\rangle_{\gamma=1}>\left\langle\pi_{i}\right\rangle_{\gamma=0}>\left\langle\pi_{i}\right\rangle_{\gamma=-1}
$$

that for $p=0$ the quenched payoffs (93) will be strictly less than the mean self-interested payoffs

$$
\left\langle\pi_{i}\right\rangle_{\gamma=0}
$$

for each $i=1,2$. Likewise, for $p=1$ the quenched payoffs (93) will be strictly greater than the mean self-interested payoffs (95) for each $i=1,2$.

Therefore, for each agent $i$ there is a critical probability for a given inverse-temperature $\beta$, $p_{i, \beta}^{*}$, such that $p>p_{i, \beta}^{*}$ implies that the quenched payoff for agent $i$ resulting from gratitude and resentment configurations is more than the strictly self-interested payoff for agent $i$. This is illustrated in Figure 2.

Now we will consider average behavior over temperature. If agents behave with different levels of rationality, that is uniformly spread out, then we may consider the mean payoffs taken over a range of temperatures. It is assumed that agents do not behave completely randomly, and always have some degree of rationality - i.e., the temperature stays below a cutoff, $T \leq T_{m}$. The temperature-mean quenched payoff, having gratitude weight $p$, is then

$$
\widetilde{\pi_{i ; p}}:=\frac{1}{T_{m}} \int_{0}^{T_{m}} \overline{\pi_{i ; p}} d T,
$$




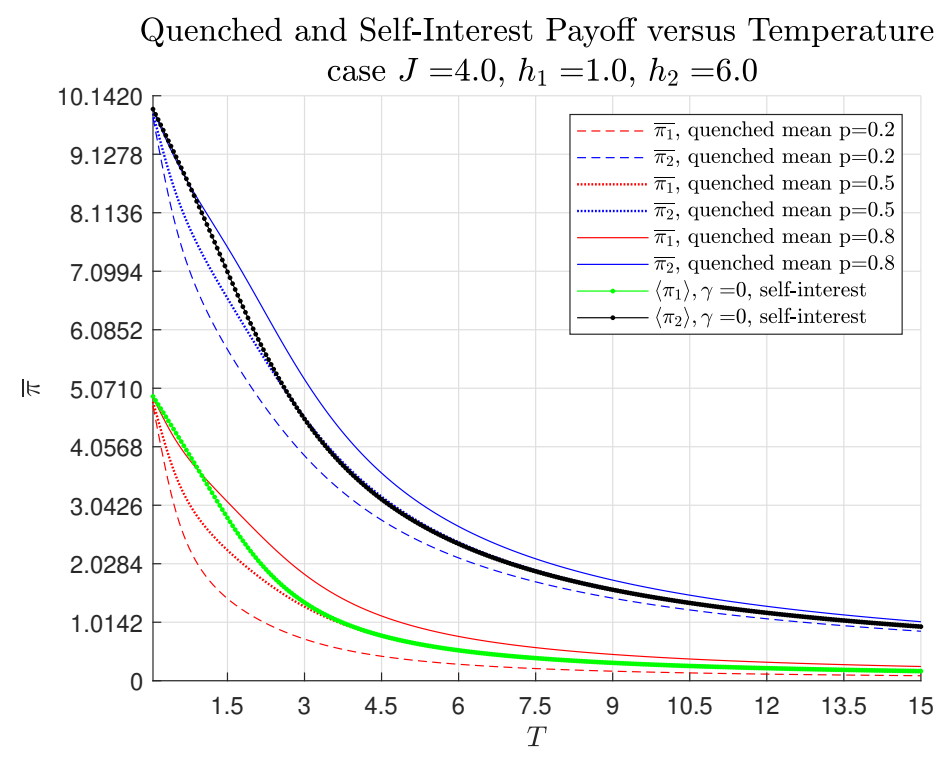

Figure 2: Two-person quenched game with self-interested payoffs and various quenched payoffs. For any fixed temperature, the quenched payoff will strictly increase with $p$, the quenched probability weight on gratitude. Therefore, the quenched payoff will be larger than the self-interest payoff when the quenched probability weight is larger than a critical value which depends on temperature and agent. Above this critical value, an agent is motivated to change from acting self-interestedly and to engage the other agent in a relationship - i.e., to engage in actions that can result in gratitude or resentment in the other agent.

where $\pi_{i ; p}$ denotes the quenched payoff with gratitude weight $p$ and resentment weight $1-p$, and we note that this quenched payoff depends on temperature $T=1 / \beta$. Likewise, denote the temperature-mean of self-interested payoff as

$$
\tilde{\pi}_{i}:=\frac{1}{T_{m}} \int_{0}^{T_{m}}\left\langle\pi_{i}\right\rangle_{\gamma=0} d T .
$$

The temperature-means of the quenched payoffs and self-interested payoffs are shown in Figure 3, assuming uniform distribution of temperature in the interval $0 \leq T \leq 15$, which is an arbitrary choice of upper bound for illustrative purposes. Consequently, we assume here that agents are not more non-rational that $T=T_{m}=15$, and that agents are equally likely to have degrees of non-rationality between zero and fifteen. Each agent has a critical quenched probability (for gratitude) above which they are motivated to engage in actions that result in gratitude or resentment from the other agent $(\gamma= \pm 1)$ rather than behave in a purely self-interested manner $(\gamma=0)$.

These critical quenched probabilities occur where the graphs in Figure 3 intersect. The critical quenched probability for agent 1 is $p_{1}^{*} \approx 0.597$, and that for agent 2 is $p_{2}^{*} \approx 0.553$.

Note that since $p_{i}^{*}>0.5$ for $i=1,2$, the agents are both averse to resentful behavior. They will not disengage from purely self-interested behavior until, on (quenched) average there is more gratitude among the two agents than resentment. This is consistent with Axiom 4 in [1] (c.f. page 73), which states that people experience an asymmetrical change between feeling 
something good and feeling something bad, being more averse to the bad than welcoming of the good. We also point out that agent 1 , who has lower payoff than agent 2 , is more averse to resentful behavior than agent 1 since agent 1 's critical probability $p_{1}^{*}$ is larger than agent 2 's critical probability $p_{2}^{*}$.

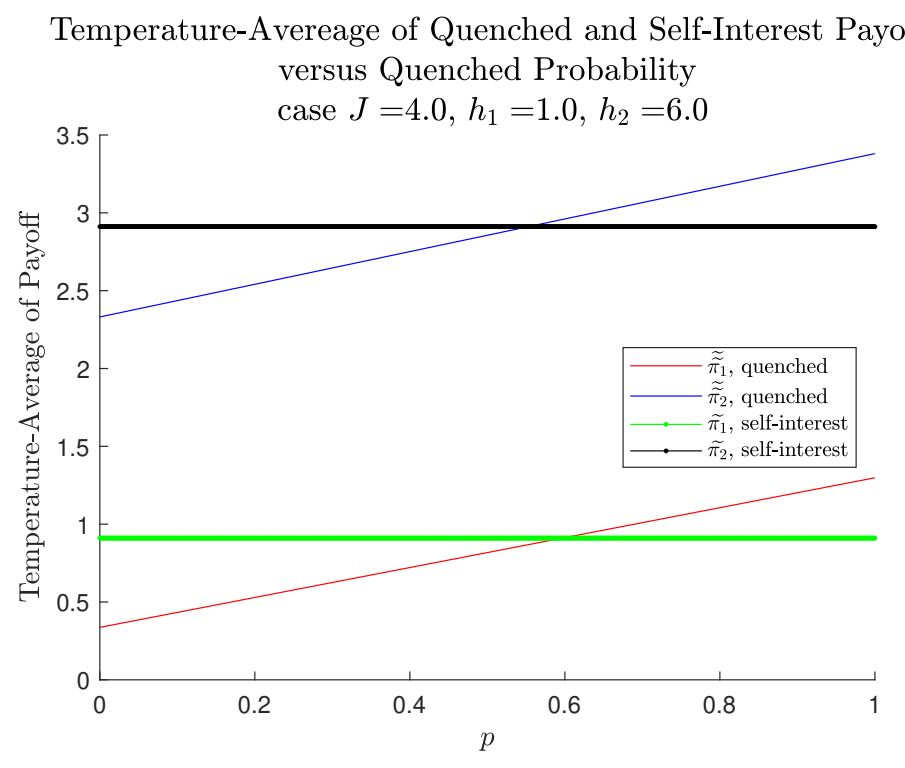

Figure 3: Temperature-Means of self-interested payoffs and quenched payoffs for a two-person quenched game as functions of quenched probability of mutual gratitude. Agents are assumed to have a uniform distribution of non-rationality degrees over $0 \leq T \leq 15$ for illustration. The quenched payoff will be larger than the self-interest payoff when the quenched probability weight for agent 1 is larger than $p_{1}^{*} \approx 0.597$. The same holds for agent 2 when their quenched probability weight is larger than $p_{2}^{*} \approx 0.553$. Above the agent's critical value, the agent is motivated on average (for all temperatures) to change from acting self-interestedly and to engage the other agent in a relationship - i.e., to engage in actions that can result in gratitude or resentment in the other agent. Both agents have critical quenched probabilities greater than one-half, indicating that they are averse to resentment, consistent with Axiom 4 in[1]. It is also evident that agent 1 , who receives less payoff, is more averse to resentment than agent 1 since $p_{1}^{*}>p_{2}^{*}$. Effort costs to switch from self-interested behavior to interactive behavior (gratitude/resentment outcomes that risk punishment) effectively lower the quenched curves and shift the intersections to the right, making agents even more resent-averse.

If we consider the more rational region $0 \leq T \leq T_{m}$ with lower non-rational cutoff temperature $T_{m}=5$, both agents are even more averse to resentful behavior. Their critical quenched probabilities for this range of temperatures are $p_{1}^{*} \approx 0.646$ and $p_{2}^{*} \approx 0.592$.

The quenched probability $p$ can be thought of as opportunities for one agent to take a costly action that yields a benefit (good) for the other agent who rewards the action (the other agent always rewards the action for this model). The cost never outweighs the reward as perceived by the participants: i.e., they will only change behavior if $p>p_{i}^{*}$.

For this particular model, the Nash equilibrium has no predictive power of Humanomics properties, as is evident from the observation in Section 7.1.1 that the difference in payoff in the gratitude state and resentment state is zero at zero temperature. As a result, the quenched and self-interested payoffs are the same for each agent and therefore neither agent is motivated to prefer self-interested behavior or to engage in a relationship with the other agent; i.e., to risk 
reward or punishment by taking actions toward the other agent that can result in mutual gratitude or resentment.

\section{Conclusion}

We have introduced simple, tractable models for which some fundamental elements in $\mathrm{Hu}$ manomics [1] were implemented. The model was built on the principle of mutual gratitude and mutual resentment intermingled with reward and punishment in the form of higher or lower payoffs. Bounded rationality is accounted for in this model, consistent with Humanomics. The additional question of timescales was also examined: there is one timescale over which gratitude and resentment change, and another over which economic equilibrium occurs. Two timescale scenarios were investigated - one where economic equilibrium occurs so quickly that gratitude and resentment are effectively constant (slow-changing) and another where economic equilibrium occurs so slowly that economic variables are effectively constant during the formation of gratitude and resentment. These two scenarios are quenched and annealed models, respectively, as in statistical mechanics. We did not examine the situation when the two timescales are the same; i.e., when economic variables and gratitude/resentment variables interact. This would be a very interesting case to examine.

The two-agent quenched aligning model in Section 7 yielded a new insight into critical quenched probabilities. There is a quenched probability of mutual gratitude $p$, which is the empirical frequency of mutual gratitude between the agents. Think of these frequencies with which opportunities arise in the environment for one agent to take action that creates gratitude in the other. This probability has a critical value $p_{i}^{*}$ for each agent $(i=1,2)$ such that agent $i$ will choose self-interestedly when $p<p_{i}^{*}$ and agent $i$ will choose to interact with the other agent, i.e. to take actions that result in gratitude/resentment and thus reward/punishment, when $p>p_{i}^{*}$.

The critical probabilities $p_{i}^{*}$ were shown to be greater than one-half, which implies that agents are only motivated to interact with each other with gratitude/resentment if there is a higher frequency of mutual gratitude than mutual resentment. This was not built into the model, but was a result of its properties. Furthermore, this is a very nice feature, since it is consistent with Axiom 4 in Humanomics about the asymmetry of gratitude and resentment: people are more averse to resentment than accepting of gratitude.

Although the Nash equilibrium was used to justify important conclusions, we showed for a particular model that the Nash equilibrium has no predictive power of Humanomics properties since the rewards are the same for self-interested behavior and for interactive behavior for said model. It is evident from this that the assumption of bounded rationality is essential in this case, and that the resulting Gibbs equilibrium does indeed lead to richer properties.

The model extends to any number of agents, and for a statistically large number of agents, the infinite-agent limit results in a spin-glass model with a discrete quenched probability distribution. Unlike a standard spin glass model in physics, this model is not frustrated and the disorder comes from high-temperature paramagnetic (random) behavior. This would be a very interesting problem as well. More work could also be done examining anti-aligning (anti-ferromagnetic) models. Correlation inequalities could still hold for a two-agent anti-aligning model by transforming it to an aligning (ferromagnetic) model. This transformation could be extended to more agents if interaction among agents could be modeled as nearest-neighbor on a rectangular lattice (like a chess board, and negating the black squares only), but this would not generalize to Curie-Weiss-type interactions among all agents. For such a case, other techniques would have to be developed. Additional research could be done on an aligning model with a low number of 
agents, $N=3,4,5,6$ to see if the results here extend to more than two agents. Finally, research on a model with heterogeneous gratitude configurations would be interesting, where one-sided gratitude is possible, and with gratitude weights that can depend on agents.

\section{Appendix A. Correlation Inequalities}

Here we show two techniques to prove positivity of correlation functions: cluster expansion and marginal FKG techniques. In particular, we want to show that the correlation function

$$
\begin{aligned}
\frac{\partial}{\partial \gamma_{m n}}\left\langle\pi_{i}\right\rangle_{\gamma} & =\frac{\partial}{\partial \gamma_{m n}} \frac{\int_{\Phi_{\Lambda}} \exp [\beta V(\mathbf{x} ; \gamma)] \pi_{i}(\mathbf{x}) d \mathbf{x}}{\mathcal{Z}_{\gamma}} \\
& =\beta\left\langle\pi_{i} \frac{\partial V}{\partial \gamma_{m n}}\right\rangle_{\gamma}-\beta\left\langle\pi_{i}\right\rangle_{\gamma}\left\langle\frac{\partial V}{\partial \gamma_{m n}}\right\rangle_{\gamma},
\end{aligned}
$$

in (59) is positive for aligning/ferromagnetic interactions; i.e., all $J_{i j} \geq 0, h_{i} \geq 0$, and $1+\gamma_{i j} \geq 0$. The payoffs $\pi_{i}$ used here are then of the form in Example 7, but we point out that more general aligning/ferromagnetic payoffs with a potential can be accommodated.

\section{Appendix A.1. Cluster Expansion}

The cluster expansion technique involves expanding the correlation function in a Taylor series. To accommodate the analysis of correlations, we "duplicate" the variables $x_{i}$ in (1) with

$$
y_{i} \in F:=[\underline{x}, \bar{x}] .
$$

The correlation in (A.2) can then be written

$$
\begin{aligned}
\left\langle\pi_{i} \frac{\partial V}{\partial \gamma_{m n}}\right\rangle_{\gamma} & -\left\langle\pi_{i}\right\rangle_{\gamma}\left\langle\frac{\partial V}{\partial \gamma_{m n}}\right\rangle_{\gamma} \\
& =\frac{1}{2 Z_{\gamma}^{2}} \iint_{F_{(2 N)}} \mathrm{e}^{\beta V(\mathbf{x} ; \gamma)+\beta V(\mathbf{y} ; \gamma)}\left[\pi_{i}(\mathbf{x})-\pi_{i}(\mathbf{y})\right]\left[\frac{\partial V}{\partial \gamma_{m n}}(\mathbf{x})-\frac{\partial V}{\partial \gamma_{m n}}(\mathbf{y})\right] d \mathbf{x} d \mathbf{y}
\end{aligned}
$$

For correlations, it will be easier to rotate variables from $\left(x_{i}, y_{i}\right) \in F^{2}$ to $\left(u_{i}, v_{i}\right) \in D_{i}$, where for the agents $i, 1 \leq i \leq N$,

$$
u_{i}=\left(x_{i}+y_{i}\right) / 2, \quad v_{i}=\left(x_{i}-y_{i}\right) / 2,
$$

and the rotated duplicated variables are in the diamond set

$$
D_{i}=D:=\left\{\left\|\left(u_{i}, v_{i}\right)\right\|_{1}=\left|u_{i}\right|+\left|v_{i}\right| \leq \bar{x}\right\} .
$$

The full rotated, duplicated configuration space is then

$$
\Upsilon_{\Lambda}:=\prod_{i \in \Lambda} D_{i}=D^{N},
$$


and the combined potentials $V(\mathbf{x} ; \gamma)+V(\mathbf{y} ; \gamma)$ in (A.4) become

$$
\begin{aligned}
V(\mathbf{u} ; \gamma)+V(\mathbf{v} ; \gamma)= & \frac{2}{N} \sum_{j<k=1}^{N}\left(1+\gamma_{j k}\right) J_{j k}\left(u_{j} u_{k}+v_{j} v_{k}\right) \\
& +\frac{2}{N} \sum_{j=1}^{N} J_{j j}\left(u_{j}^{2}+v_{j}^{2}\right)+2 \sum_{j=1}^{N} h_{j} u_{j} .
\end{aligned}
$$

The difference functions in (A.4) have rotated forms

$$
\pi_{i}(\mathbf{u})-\pi_{i}(\mathbf{v})=\frac{2}{N} \sum_{m \leq i=1}^{N} J_{m i}\left(u_{m} v_{i}+u_{i} v_{m}\right)+\frac{2}{N} \sum_{m>i=1}^{N} J_{i m}\left(u_{m} v_{i}+u_{i} v_{m}\right)+2 h_{i} v_{i},
$$

and

$$
\frac{\partial V}{\partial \gamma_{m n}}(\mathbf{u} ; \gamma)-\frac{\partial V}{\partial \gamma_{m n}}(\mathbf{v} ; \gamma)=\frac{2}{N} \sum_{j<k=1}^{N} J_{m n}\left(u_{j} v_{k}+u_{k} v_{j}\right) .
$$

We point out that all terms in (A.8), (A.9), and (A.10) have nonnegative coefficients.

The exponential in (A.4) can be expanded in a Taylor series to yield

$$
\begin{aligned}
& \left\langle\pi_{i} \frac{\partial V}{\partial \gamma_{m n}}\right\rangle_{\gamma}-\left\langle\pi_{i}\right\rangle_{\gamma}\left\langle\frac{\partial V}{\partial \gamma_{m n}}\right\rangle_{\gamma} \\
& \quad=\sum_{r=0}^{\infty} \frac{\beta^{r}}{2 r ! \mathcal{Z}_{\gamma}^{2}} \iint_{D^{N}}[V(\mathbf{u} ; \gamma)+V(\mathbf{v} ; \gamma)]^{r}\left[\pi_{i}(\mathbf{u})-\pi_{i}(\mathbf{v})\right]\left[\frac{\partial V}{\partial \gamma_{m n}}(\mathbf{u})-\frac{\partial V}{\partial \gamma_{m n}}(\mathbf{v})\right] d \mathbf{u} d \mathbf{v},
\end{aligned}
$$

and since $\beta \geq 0$, it only needs to be shown that the integrals in (A.11) are positive. To that end, an idea from graphical representations of continuous spin models will be used [34, 35]. We represent each continuous "spin" $u_{i}$ and $v_{i}$ as an Ising \pm 1 spin multiplied by an interaction,

$$
\begin{aligned}
& u_{i}=\sigma_{i} s_{i}, \\
& \sigma_{i}= \pm 1, \quad s_{i}=\left|u_{i}\right|, \\
& v_{i}=\tau_{i} t_{i}, \\
& \tau_{i}= \pm 1, \quad t_{i}=\left|v_{i}\right|,
\end{aligned}
$$

where the interactions are integrated over the positive quadrant of the diamond (A.6)

$$
D^{+}:=\left\{\left(s_{i}, t_{i}\right) \mid s_{i} \geq 0, t_{i} \geq 0,\left\|\left(s_{i}, t_{i}\right)\right\|_{1} \leq \bar{x}\right\} .
$$

Configurations of the graphical representation variables are denoted

$$
\begin{aligned}
\vec{\sigma} & :=\left(\sigma_{1}, \sigma_{2}, \ldots, \sigma_{N}\right) \in\{-1,+1\}^{N}, \\
\vec{s} & :=\left(s_{1}, s_{2}, \ldots, s_{N}\right) \\
\vec{\tau} & :=\left(\tau_{1}, \tau_{2}, \ldots, \tau_{N}\right) \in\{-1,+1\}^{N}, \\
\vec{t} & :=\left(t_{1}, t_{2}, \ldots, t_{N}\right),
\end{aligned}
$$

where

$$
(\vec{s}, \vec{t}):=\prod_{i=1}^{N}\left(s_{i}, t_{i}\right) \in\left(D^{+}\right)^{N}
$$


The integrals in (A.11) have the following form in the graphical representation variables:

$$
\iint_{\left(D^{+}\right)^{N}} d \vec{s} d \vec{t} \sum_{\vec{\sigma} \in\{-1,1\}^{N}} \sum_{\vec{\tau} \in\{-1,1\}^{N}} \prod_{i_{1}} \sigma_{i_{1}} \prod_{i_{2}} \tau_{i_{2}} \prod_{i_{3}} s_{i_{3}}^{m_{i_{3}}} \prod_{i_{4}} t_{i_{4}}^{n_{i_{4}}} .
$$

If a single spin $\sigma_{i}$ or $\tau_{i}$ appears in the product (A.20), summing over $\sigma_{i}= \pm 1, \tau_{i}= \pm 1$ will result in a value of zero. If the spin products are empty (i.e., equal to one), then the result is an integral of the variables $s_{i}$ and $t_{i}$ to various powers. Since the $s_{i}$ and $t_{i}$ on nonnegative on their domain $\left(D^{+}\right)^{N}$, we see that the integral in (A.20) is positive, and thus the correlation in (A.11) is positive. Note that this result requires nonnegativity of the terms $\left(1+\gamma_{m n}\right)$. This allows the resentment value for $\gamma_{m n}$ to be as low as -1 .

As a result, from (59) it is seen that the derivative of any agent's expected payoff function $\left\langle\pi_{i}\right\rangle_{\gamma}$ is strictly increasing in the gratitude variables $\gamma_{m i}$. Clearly, if agent $m$ adds to their action function the payoff of another agent $i$ and agent $i$ adds to their action function the payoff of agent $m$ (which is effectively changing $\gamma_{m i}=\gamma_{i m}$ from 0 to 1 ), then the expected payoff of agent $i$ with respect to the Gibbs measure will increase, as will the expected payoff of agent $m$. The requirement for a potential restricts this scenario to mutually reciprocated beneficence, as opposed to the one-way beneficence in Example 5. This was previously discussed in Example 2. In any case, the increase in payoffs for agents $i$ and $m$ demonstrates that adding payoffs to actions is mutually beneficial to both agents, which we describe as "mutual beneficence". We mention the caveat that the condition of Case 1 in the Desert Lemma of [1] that agent $m$ did not have to add to their action the payoff of agent $i$, is not clear here. Rather the act of adding payoffs is mutual between agent $i$ and agent $m$, more directly in accord with Principle 7 (page 78 [1]):

An equilibrium concept, if it can be said to exist, is based on mutual fellow-feeling and lives in rule space.

The appropriate way to interpret this section is as follows.

$\star$ The rule of mutual beneficence is encoded into the requirements for a potential in this scenario.

That mutual addition of payoffs is interpreted as mutual beneficence is a result of the positivity of correlation functions in (A.2).

\section{Appendix A.2. FKG Measures}

As seen in Example 6, agents may need to add functions other than the payoff function to their actions to increase the mean payoff of other agents. Accordingly, we present another method to show positivity of the correlation function (A.2) using a more general method than the cluster expansion technique in Subsection Appendix A.1. First, we write the partition function (57) with the variables in (A.12),

$$
\mathcal{Z}_{\gamma}=\int_{\Phi_{\Lambda}} \exp [\beta V(\mathbf{x} ; \gamma)] d \mathbf{x}=\int_{\vec{u} \in[0, \bar{x}]^{N}} d \vec{u} \sum_{\vec{\sigma} \in\{-1,1\}^{N}} \exp [\beta V(\vec{\sigma}, \vec{u})] .
$$

where for all $i$, we decompose each strategy variable as a product of an Ising spin and a magnitude:

$$
\begin{aligned}
& x_{i}=\sigma_{i} u_{i}, \\
& \sigma_{i}= \pm 1, \quad u_{i}=\left|x_{i}\right| \\
& \vec{\sigma}=\left(\sigma_{1}, \sigma_{2}, \ldots, \sigma_{N}\right) \in\{-1,1\}^{N}, \\
& \vec{u}=\left(u_{1}, u_{2}, \ldots, u_{N}\right) \in[0, \bar{x}]^{N} .
\end{aligned}
$$


We define the Ising partition function as

$$
Z_{\vec{u}}^{I}=\sum_{\vec{\sigma} \in\{-1,1\}^{N}} \exp [\beta V(\vec{\sigma}, \vec{u})] .
$$

The corresponding Ising measure defined on functions $f$ of $\vec{\sigma}$ is

$$
\rho_{\vec{u}}^{I}(f):=\frac{1}{\mathcal{Z}_{\vec{u}}^{l}} \sum_{\vec{\sigma} \in\{-1,1\}^{N}} f(\vec{\sigma}) \exp [\beta V(\vec{\sigma}, \vec{u})] .
$$

For the next well-known result, we need that spin configurations form a lattice with a natural partial ordering. For two configurations $\vec{\sigma}^{a}$ and $\vec{\sigma}^{b}, \vec{\sigma}^{a} \geq \vec{\sigma}^{b}$ if and only if $\sigma_{i}^{a} \geq \sigma_{i}^{b}$ for all $i$, $1 \leq i \leq N$. We denote the maximum configuration as $\vec{\sigma}^{a} \vee \vec{\sigma}^{b}$ and the minimum configuration $\vec{\sigma}^{a} \wedge \vec{\sigma}^{b}$ by

$$
\begin{aligned}
& \left(\vec{\sigma}^{a} \vee \vec{\sigma}^{b}\right)_{i}:=\max \left\{\sigma_{i}^{a}, \sigma_{i}^{b}\right\}, \\
& \left(\vec{\sigma}^{a} \wedge \vec{\sigma}^{b}\right)_{i}:=\min \left\{\sigma_{i}^{a}, \sigma_{i}^{b}\right\} .
\end{aligned}
$$

We say a function $f$ on $\{-1,1\}^{N}$ is increasing if $\vec{\sigma}^{a} \geq \vec{\sigma}^{b}$ implies $f\left(\vec{\sigma}^{a}\right) \geq f\left(\vec{\sigma}^{b}\right)$.

Proposition 2 ( FKG Inequality for Ising Measure [36] ). The Ising measure defined in (A.24) satisfies the FKG lattice condition for any configuration $\vec{u}$. That is, given two spin configurations $\vec{\sigma}^{a}, \vec{\sigma}^{b} \in\{-1,1\}^{N}$,

$$
\rho_{\vec{u}}^{I}\left(\vec{\sigma}^{a} \vee \vec{\sigma}^{b}\right) \rho_{\vec{u}}^{I}\left(\vec{\sigma}^{a} \wedge \vec{\sigma}^{b}\right) \geq \rho_{\vec{u}}^{I}\left(\vec{\sigma}^{a}\right) \rho_{\vec{u}}^{I}\left(\vec{\sigma}^{b}\right) .
$$

Therefore for two increasing functions $f$ and $g$ on $\{-1,1\}^{N}$, the FKG inequality holds:

$$
\rho_{\vec{u}}^{I}(f g) \geq \rho_{\vec{u}}^{I}(f) \rho_{\vec{u}}^{I}(g) .
$$

Theorem 2.6 of [37] shows that (A.26) only needs to be checked for configurations that differ only at two sites. The condition (A.26) can then be shown directly to hold for the measure in (A.24). It will be useful to show that the marginal measure on the $\vec{u}$ also satisfies the FKG lattice condition. For a continuous function $H(\vec{u})$ on the positive cone of configuration space $[0, \bar{x}]^{N}$, we define the marginal measure on spin magnitudes by

$$
\rho^{+}(H):=\frac{1}{\mathcal{Z}^{+}} \int_{[0, \bar{x}]^{N}} H(\vec{u}) \mathcal{Z}_{\vec{u}}^{I} d \vec{u},
$$

where $\mathcal{Z}^{+}$is the spin magnitude partition function given by

$$
\mathcal{Z}^{+}:=\int_{[0, \bar{x}]^{N}} \mathcal{Z}_{\vec{u}}^{I} d \vec{u} .
$$

Proposition 3 ( FKG Inequality for Magnitude Measure [34] ). The magnitude measure defined in (A.28) satisfies the FKG lattice condition. That is, given two magnitude configurations $\vec{u}^{a}, \vec{u}^{b} \in[0, \bar{x}]^{N}$,

$$
\rho^{+}\left(\vec{u}^{a} \vee \vec{u}^{b}\right) \rho^{+}\left(\vec{u}^{a} \wedge \vec{u}^{b}\right) \geq \rho^{+}\left(\vec{u}^{a}\right) \rho^{+}\left(\vec{u}^{b}\right) .
$$

Therefore for two increasing functions $f$ and $g$ on $[0, \bar{x}]^{N}$, the FKG inequality holds:

$$
\rho^{+}(f g) \geq \rho^{+}(f) \rho^{+}(g) \text {. }
$$


Proof (1). Once again, theorem 2.6 of [37] shows that (A.26) only needs to be checked for configurations that differ only at two sites. Let $c, d \in[0, \bar{x})$ and choose $\delta>0$ such that $c+\delta, d+\delta \in$ $[0, \bar{x}]$. Assume $\vec{u}^{a}$ and $\vec{u}^{b}$ differ only at sites $m$ and $n$, and that

$$
\begin{aligned}
\vec{u}_{m}^{a} & =c+\delta, & \vec{u}_{n}^{a} & =d, \\
\vec{u}_{m}^{b} & =c, & \vec{u}_{n}^{b} & =d+\delta, \\
\left(\vec{u}^{a} \vee \vec{u}^{b}\right)_{m} & =c+\delta, & \left(\vec{u}^{a} \vee \vec{u}^{b}\right)_{n} & =d+\delta, \\
\left(\vec{u}^{a} \wedge \vec{u}^{b}\right)_{m} & =c, & \left(\vec{u}^{a} \wedge \vec{u}^{b}\right)_{n} & =d .
\end{aligned}
$$

We now use the ideas in [34]. The FKG lattice condition in (A.30) then amounts to proving

$$
\frac{\mathcal{Z}_{\vec{u}}^{I}(c+\delta, d+\delta)}{\mathcal{Z}_{\vec{u}}^{I}(c, d+\delta)} \geq \frac{\mathcal{Z}_{\vec{u}}^{I}(c+\delta, d)}{\mathcal{Z}_{\vec{u}}^{I}(c, d)},
$$

where $\mathcal{Z}_{\vec{u}}^{I}\left(u_{m}, u_{n}\right)$ is the value of the Ising partition function in (A.23) with the values at sites $m$ and $n$ indicated explicitly. To prove (A.33), it suffices to show

$$
\frac{\partial}{\partial d} \frac{\mathcal{Z}_{\vec{u}}^{I}(c+\delta, d)}{\mathcal{Z}_{\vec{u}}^{I}(c, d)} \geq 0,
$$

which, upon taking the derivative, is equivalent to showing

$$
\frac{\frac{\partial}{\partial d} \mathcal{Z}_{\vec{u}}^{I}(c+\delta, d)}{\mathcal{Z}_{\vec{u}}^{I}(c+\delta, d)} \geq \frac{\frac{\partial}{\partial d} \mathcal{Z}_{\vec{u}}^{I}(c, d)}{\mathcal{Z}_{\vec{u}}^{I}(c, d)}
$$

To show (A.35), it suffices that

$$
\frac{\partial}{\partial c} \frac{\frac{\partial}{\partial d} \mathcal{Z}_{\vec{u}}^{I}(c, d)}{\mathcal{Z}_{\vec{u}}^{I}(c, d)} \geq 0,
$$

which, from the derivative, is equivalent to showing

$$
\mathcal{Z}_{\vec{u}}^{I}(c, d)\left[\frac{\partial}{\partial c} \frac{\partial}{\partial d} \mathcal{Z}_{\vec{u}}^{I}(c, d)\right]-\left[\frac{\partial}{\partial c} \mathcal{Z}_{\vec{u}}^{I}(c, d)\right]\left[\frac{\partial}{\partial d} \mathcal{Z}_{\vec{u}}^{I}(c, d)\right] \geq 0 .
$$

Rearranging (A.37), and recalling that $(c, d)$ represents the values for $\left(u_{m}, u_{n}\right)$, we see that it is enough to prove

$$
\frac{\frac{\partial}{\partial u_{m}} \frac{\partial}{\partial u_{m}} \mathcal{Z}_{\vec{u}}^{I}}{\mathcal{Z}_{\vec{u}}^{I}} \geq \frac{\frac{\partial}{\partial u_{m}} \mathcal{Z}_{\vec{u}}^{I}}{\mathcal{Z}_{\vec{u}}^{I}} \cdot \frac{\frac{\partial}{\partial u_{n}} \mathcal{Z}_{\vec{u}}^{I}}{\mathcal{Z}_{\vec{u}}^{I}}
$$

Since the $J_{i j} \geq 0, h_{i} \geq 0$, and $u_{i} \geq 0$ for all $i, j$, (A.38) will hold if the Ising spin measure satisfies

$$
\begin{aligned}
\rho_{\vec{u}}^{I}\left(\sigma_{m} \sigma_{n}\right) & \geq \rho_{\vec{u}}^{I}\left(\sigma_{m}\right) \cdot \rho_{\vec{u}}^{I}\left(\sigma_{n}\right) \geq 0, \\
\rho_{\vec{u}}^{I}\left(\sigma_{i} \sigma_{m} \sigma_{j}\right) & \geq \rho_{\vec{u}}^{I}\left(\sigma_{i} \sigma_{m}\right) \cdot \rho_{\vec{u}}^{I}\left(\sigma_{j}\right), \\
\rho_{\vec{u}}^{I}\left(\sigma_{i} \sigma_{m} \sigma_{j} \sigma_{n}\right) & \geq \rho_{\vec{u}}^{I}\left(\sigma_{i} \sigma_{m}\right) \cdot \rho_{\vec{u}}^{I}\left(\sigma_{j} \sigma_{n}\right),
\end{aligned}
$$

for all $i, j, m, n \in \Lambda$. The proofs of (A.39a), (A.39b), and (A.39c) are shown in [38]. 
Proof (2). We will also outline an approach that may work for more general situations. We point out that both proofs require ferromagnetic systems: $\left(1+\gamma_{i j}\right) J_{i j} \geq 0$ for all $i, j \in \Lambda$, as well as all $h_{i} \geq 0$. This proof picks up at proving (A.39a), (A.39b), and (A.39c).

A random cluster measure can be constructed as in [37] (chapter 1, section 4). Because we have a non-uniform field of $h_{i} \geq 0$, we add an additional site $\oplus$ to $\Lambda$ with spin $\sigma_{\oplus}$ that has fixed value +1 , giving the new set of sites $\Lambda_{\oplus}$. This results in a cluster, the one connected to site $\oplus$, having spins that are forced to all have the value +1 . Since the $\oplus$-cluster spins can only have one value instead of two values, we must subtract one from the exponent of the cluster weight $k(\omega)$, where $k(\omega)$ denotes the number of connected components in the graph of the configuration $\omega$. We also point out that the probabilities are not uniform, but depend on the $J_{i j} \geq 0$.

Some notation is needed. Let $G=\left(\Lambda_{\oplus}, E\right)$ be the graph of vertices $\Lambda_{\oplus}$ and edges $E=$ $\left\{\langle i, j\rangle \mid i, j \in \Lambda_{\oplus}\right\}$. Denote the spin configurations by $\Sigma=\{-1,1\}^{\Lambda}$ and the edge configurations by $\Omega=\{0,1\}^{E}=\{\omega: E \rightarrow\{0,1\}\}$.

The random cluster measure $\phi_{\vec{p}, 2}$ in [37] (chapter 1.2, equation 1.1) can be generalized to non-uniform probabilities $p_{i j}$ determined by the interactions $\beta\left(1+\gamma_{i j}\right) J_{i j}(i, j \in \Lambda)$ and $\beta h_{i}$ $(i \in \Lambda, j=\oplus)$ of the potential $V(\vec{\sigma}, \vec{u})$. The probability distribution function $\mu(\vec{\sigma}, \omega)$ on $\Sigma \times \Omega$ (defined in [37], chapter 1.4, equation 1.8) has $\vec{\sigma}$-marginal $\rho_{\vec{u}}^{I}$ and $\omega$-marginal $\phi_{\vec{p}, 2}$ (c.f., [37] theorem 1.10).

By setting the spin $\sigma_{n}$ in (A.39c) to $\sigma_{\oplus} \equiv+1$ we get (A.39b), and by setting the spins $\sigma_{i}=\sigma_{j}=\sigma_{\oplus}$ we get the left inequality in (A.39a). It is then sufficient to prove (A.39c) for $i, j, m, n \in \Lambda_{\oplus}$ and that for any $i \in \Lambda$,

$$
\rho_{\vec{u}}^{I}\left(\sigma_{i}\right)=\rho_{\vec{u}}^{I}\left(\sigma_{i} \sigma_{\oplus}\right) \geq 0 .
$$

The proof of (A.40) is a minor modification of theorem 1.16 in [37], accounting for non-uniform bond probabilities and a single-valued spin $\sigma_{\oplus}$, that relates this two-point function to the probability that two sites are in the same cluster. To prove (A.39c), we use the identity

$$
\sigma_{i} \sigma_{m}=2 \delta_{\sigma_{i}, \sigma_{m}}-1,
$$

where the Kronecker delta function $\delta_{a, b}=1$ if $a=b$ and $\delta_{a, b}=0$ if $a \neq b$. It then suffices to show

$$
\rho_{\vec{u}}^{I}\left(\delta_{\sigma_{i}, \sigma_{m}} \delta_{\sigma_{j}, \sigma_{n}}\right) \geq \rho_{\vec{u}}^{I}\left(\delta_{\sigma_{i}, \sigma_{m}}\right) \rho_{\vec{u}}^{I}\left(\delta_{\sigma_{j}, \sigma_{n}}\right)
$$

for all $i, j, m, n \in \Lambda_{\oplus}$. Note that the probability weight for spin configuration $\vec{\sigma}$ from the measure $\rho_{\vec{u}}^{I}$ can be written $\rho_{\vec{u}}^{I}\left(\mathbb{I}_{\vec{\sigma}}\right)$, where $\mathbb{I}_{\vec{\sigma}}$ is the indicator function of the single configuration $\vec{\sigma}$ on $\Sigma$. Using the ideas in the proof of theorem 1.16 in [37], we have

$$
\begin{aligned}
\rho_{\vec{u}}^{I}\left(\delta_{\sigma_{i}, \sigma_{m}} \delta_{\sigma_{j}, \sigma_{n}}\right) & =\sum_{\vec{\sigma}} \delta_{\sigma_{i}, \sigma_{m}} \delta_{\sigma_{j}, \sigma_{n}} \rho_{\vec{u}}^{I}\left(\mathbb{I}_{\vec{\sigma}}\right) \\
& =\sum_{\vec{\sigma}} \delta_{\sigma_{i}, \sigma_{m}} \delta_{\sigma_{j}, \sigma_{n}}\left(\sum_{\omega} \mu(\vec{\sigma}, \omega)\right) \\
& =\sum_{\omega}\left(\sum_{\vec{\sigma}} \delta_{\sigma_{i}, \sigma_{m}} \delta_{\sigma_{j}, \sigma_{n}} \mu(\vec{\sigma} \mid \omega)\right) \phi_{\vec{p}, 2}\left(\mathbb{I}_{\omega}\right) .
\end{aligned}
$$

We claim that the summand in (A.43) satisfies

$$
\sum_{\vec{\sigma}} \delta_{\sigma_{i}, \sigma_{m}} \delta_{\sigma_{j}, \sigma_{n}} \mu(\vec{\sigma} \mid \omega) \geq\left[\frac{1}{2} \mathbb{I}_{i \leftrightarrow m}(\omega)+\mathbb{I}_{i \leftrightarrow m}(\omega)\right]\left[\frac{1}{2} \mathbb{I}_{j \leftrightarrow n}(\omega)+\mathbb{I}_{j \leftrightarrow n}(\omega)\right] .
$$


We outline the case of a configuration corresponding to the 'disconnected' terms in (A.44); i.e., assume $\omega$ is an edge configuration such that $i \leftrightarrow m$ ( $i$ is not in the same cluster as $m$ ) and $j \leftrightarrow n$. There are four possibilities for $\omega$ :
(a) $i \leftrightarrow j, m \leftrightarrow n$,
(b) $i \leftrightarrow j, m \leftrightarrow n$,
(c) $i \leftrightarrow j, m \leftrightarrow n$,
(d) $i \leftrightarrow j, m \leftrightarrow n$

In the random-cluster configurations, all clusters are composed of spins having the same value. Thus we can use the notation of a sum over a cluster having value +1 or -1 , which represents the value of all spins in that cluster. For example, if $i$ and $j$ are in the same cluster $C_{i j}$ and $m$ and $n$ are in the same cluster $C_{m n} \neq C_{i j}$, we can write

$$
\sum_{\vec{\sigma}} \delta_{\sigma_{i}, \sigma_{m}} \delta_{\sigma_{j}, \sigma_{n}} \mu(\vec{\sigma} \mid \omega)=\frac{1}{2^{2}} \sum_{C_{i j}= \pm 1} \sum_{C_{m n}= \pm 1} \delta_{\sigma_{i}, \sigma_{m}} \delta_{\sigma_{j}, \sigma_{n}},
$$

where the denominator of the leading fraction is a result of all other clusters being summed out except the two distinct clusters $C_{i j}$, and $C_{m n}$, which have $2^{2}$ possible values between them. Using this notation, the cases in a through d above, when $i, j, m, n \in \Lambda$, result in the values

$$
\begin{aligned}
& \text { (A) } \frac{1}{2^{2}} \sum_{C_{i j}} \sum_{C_{m n}} \delta_{\sigma_{i}, \sigma_{m}} \delta_{\sigma_{j}, \sigma_{n}}=\frac{1}{2}, \\
& \text { (B) } \frac{1}{2^{3}} \sum_{C_{i j}} \sum_{C_{m}} \sum_{C_{n}} \delta_{\sigma_{i}, \sigma_{m}} \delta_{\sigma_{j}, \sigma_{n}}=\frac{1}{4}, \\
& \text { (C) } \frac{1}{2^{3}} \sum_{C_{i}} \sum_{C_{j}} \sum_{C_{m n}} \delta_{\sigma_{i}, \sigma_{m}} \delta_{\sigma_{j}, \sigma_{n}}=\frac{1}{4}, \\
& \text { (D) } \frac{1}{2^{4}} \sum_{C_{i}} \sum_{C_{j}} \sum_{C_{m}} \sum_{C_{n}} \delta_{\sigma_{i}, \sigma_{m}} \delta_{\sigma_{j}, \sigma_{n}}=\frac{1}{4} .
\end{aligned}
$$

In all cases above for the configuration $\omega$ satisfying $i \leftrightarrow m$ and $j \leftrightarrow n$, the summed values are greater than or equal to

$$
\frac{1}{2} \mathbb{I}_{i \leftrightarrow m}(\omega) \frac{1}{2} \mathbb{I}_{j \leftrightarrow n}(\omega)=\frac{1}{4} .
$$

The other cases follow in a similar manner, even if one of the spins is at the site $\oplus$, to prove the claim (A.44). Finally, we use that the random cluster measure satisfies the FKG lattice condition on edge configurations (c.f., [37] theorem 3.8) and that the functions $\frac{1}{2} \mathbb{I}_{k \leftrightarrow l}+\mathbb{I}_{k \leftrightarrow l}$ are increasing in $\omega$ for any $l, k \in \Lambda_{\oplus}$, along with (A.43) and (A.44), to see that

$$
\begin{aligned}
\rho_{\vec{u}}^{I}\left(\delta_{\sigma_{i}, \sigma_{m}} \delta_{\sigma_{j}, \sigma_{n}}\right) & \geq \phi_{\vec{p}, 2}\left(\frac{1}{2} \mathbb{I}_{i \leftrightarrow m}(\omega)+\mathbb{I}_{i \leftrightarrow m}(\omega)\right) \phi_{\vec{p}, 2}\left(\frac{1}{2} \mathbb{I}_{i \leftrightarrow m}(\omega)+\mathbb{I}_{i \leftrightarrow m}(\omega)\right) \\
& =\rho_{\vec{u}}^{I}\left(\delta_{\sigma_{i}, \sigma_{m}}\right) \rho_{\vec{u}}^{I}\left(\delta_{\sigma_{j}, \sigma_{n}}\right) .
\end{aligned}
$$

We now prove the correlation function in (A.2) is nonnegative using FKG techniques. This amounts to proving correlation inequalities of the form

$$
\begin{aligned}
\left\langle x_{i} x_{m} x_{n}\right\rangle_{\gamma} & \geq\left\langle x_{i}\right\rangle_{\gamma}\left\langle x_{m} x_{n}\right\rangle_{\gamma}, \\
\left\langle x_{i} x_{j} x_{m} x_{n}\right\rangle_{\gamma} & \geq\left\langle x_{i} x_{j}\right\rangle_{\gamma}\left\langle x_{m} x_{n}\right\rangle_{\gamma} .
\end{aligned}
$$


We first prove (A.48b). The function $\rho_{\vec{u}}^{I}\left(\sigma_{i} \sigma_{j}\right)$ is increasing in $\vec{u}$ for any $i, j \in \Lambda$ since its derivative with respect to any $u_{k}$ results in correlation functions which are positively proportional to the difference of the left-side and right-side of (A.39b) and the same for (A.39c). The positivity of these differences was proved above as a result of (A.47). The functions $u_{i}$ are also clearly increasing in $\vec{u}$. Using the FKG properties of $\rho_{\vec{u}}^{I}$ and $\rho^{+}$, it is seen that

$$
\begin{aligned}
\left\langle x_{i} x_{j} x_{m} x_{n}\right\rangle_{\gamma} & =\rho^{+}\left(u_{i} u_{j} u_{m} u_{n} \rho_{\vec{u}}^{I}\left(\sigma_{i} \sigma_{j} \sigma_{m} \sigma_{n}\right)\right) \\
& \geq \rho^{+}\left(u_{i} u_{j} \rho_{\vec{u}}^{I}\left(\sigma_{i} \sigma_{j}\right) u_{m} u_{n} \rho_{\vec{u}}^{I}\left(\sigma_{m} \sigma_{n}\right)\right) \\
& \geq \rho^{+}\left(u_{i} u_{j} \rho_{\vec{u}}^{I}\left(\sigma_{i} \sigma_{j}\right)\right) \rho^{+}\left(u_{m} u_{n} \rho_{\vec{u}}^{I}\left(\sigma_{m} \sigma_{n}\right)\right) \\
& =\left\langle x_{i} x_{j}\right\rangle_{\gamma}\left\langle x_{m} x_{n}\right\rangle_{\gamma} .
\end{aligned}
$$

The case of (A.48a) follows the same reasoning.

It should be pointed out that for reward to occur, the correlations in (A.48a) and (A.48b) should be strictly positive, as was shown using the cluster expansion. To do this, it is enough to show a strict inequality, as was done in Proposition 3, item A, and that such a strict inequality persists on a subset of magnitude configurations of positive measure.

\section{Acknowledgments}

I, Michael Campbell, thank my daughters Sophia Campbell and Hannah Campbell for their inspiration, Shannon Starr for useful discussions about spin glasses, and of course Sabiou Inoua and Vernon Smith for their collaboration.

I, Vernon Smith, acknowledge support from Chapman University, Economic Science Institute.

We, Vernon Smith and Michael Campbell, would like to thank Sabiou Inoua for his help.

\section{References}

[1] Vernon L. Smith and Bart J. Wilson. Humanomics: Moral Sentiments and the Wealth of Nations for the Twenty-First Century. Cambridge University Press, 2019.

[2] Adam Smith. The Theory of Moral Sentiments; or, An Essay towards an Analysis of the Principles by which Men naturally judge concerning the Conduct and Character, first of their Neighbours, and afterwards of themselves. To which is added, A Dissertation on the Origins of Languages. New Edition. With a biographical and critical Memoir of the Author. By Dugald Stewart (London: Henry G. Bohn), 1853. Public domain, https://oll.libertyfund.org/titles/2620.

[3] Vernon L. Smith, Sabiou Inoua, and Michael J. Campbell. Private discussion.

[4] M. Campbell. Boundedly-rational fast-tuning control theory and statistical mechanics. Journal of Mathematical Economics and Finance, 5(1):7-18, 2019. DOI: https://doi.org/10.14505/jmef.v5.1(8).01.

[5] A. A. Cournot. Recherches sur les principes mathematiques de la theorie des richesses. 1838. public domain.

[6] M. Campbell. A Gibbsian approach to potential game theory. http://arxiv.org/abs/cond-mat/0502112, 2005.

[7] M. Campbell. Speculative and hedging interaction model in oil and U.S. dollar markets - long-term investor dynamics and phases. Physica A: Statistical Mechanics and its Applications, 540, 2020. Article 123251, https://doi.org/10.1016/j.physa.2019.123251.

[8] D. Carfì and F. Musolino. Speculative and hedging interaction model in oil and U.S. dollar markets with financial transaction taxes. Economic Modelling, 37(0):306-319, 2014. https://dx.doi.org/10.1016/j.econmod.2013.11.003.

[9] D. Carfì and M. Campbell. Bounded rational speculative and hedging interaction model in oil and U.S. dollar markets. Journal of Mathematics Economics and Finance, 1(1):4-28, 2015. ISSN 2458-0813.

[10] D. Carfì and M. Campbell. Speculative and hedging interaction model in oil and U.S. dollar markets: Nash equilibria for one or more banks. Atti della Accademia Peloritana dei Pericolanti Journal, 96(1), 2018. http://dx.doi.org/10.1478/AAPP.96S1A1. 
[11] M. Campbell and D. Carfì. Speculative and hedging interaction model in oil and U.S. dollar markets-phase transition. Journal of Statistical Physics, 170(1):165-205, 2018. https://doi.org/10.1007/s10955-017-1915-7.

[12] M. Jackson and Y. Zenou. Games on Networks, Handbook of Game Theory, volume 4. Peyton Young and Shmuel Zamir, eds., Elsevier Science, 2014.

[13] W. Weidlich and G. Haag. Concepts and Models of a Quantitative Sociology. The Dynamics of Interacting Populations. Springer Verlag, 1983.

[14] H. Föllmer. Random economies with many interacting agents. Journal of Mathematical Economics, 1:51-62, 1974.

[15] C. Hsieh, M. K onig, and X. Liu. Network formation with local complements and global substitutes: The case of r\&d networks. University of Zurich, Department of Economics, 2017. Working Paper No. 217.

[16] S. P. Anderson, J. K. Goeree, and C. A. Holt. Noisy directional learning and the logit equilibrium. The Scandinavian Journal of Economics, 106(3):581-602, 2004. doi:10.1111/j.0347-0520.2004.00378.x.

[17] S. P. Anderson, J. K. Goeree, and C. A. Holt. The logit equilibrium: A perspective on intuitive behavioral anomalies. Southern Economic Journal, 69(1):21-47, 2002.

[18] K. Binmore and L. Samuelson. Muddling through: Noisy equilibrium selection. Journal of Economic Theory, $74: 235-265,1997$.

[19] S. Grauwin, E. Bertin, R. Lemoy, and P. Jensen. Competition between collective and individual dynamics. Proceedings of the National Academy of Sciences, 106(49):20622-20626, 2009.

[20] W. Brock and S. Durlauf. Discrete choice with social interactions. Review of Economic Studies, 68(2):235-260, 2001.

[21] M. Campbell. Inevitability of collusion in a coopetitive bounded rational Cournot model with increasing demand. Journal of Mathematical Economics and Finance, 2(1):7-20, 2016. ISSN 2458-0813.

[22] M. Campbell and M. Seifert. Private discussion.

[23] D. Monderer and L. S. Shapley. Potential games. Games and Economic Behavior, 14:124-143, 1996.

[24] J. Johnson, G. J. Tellis, and D. J. Macinnis. Losers, winners, and biased trades. Journal of Consumer Research, 32(2):324-329, 2005.

[25] H. Simon. A behavioral model of rational choice. The Quarterly Journal of Economics, 69(1):99-118, 1955.

[26] W. Kang and K. Ramanan. Characterization of stationary distributions of reflected diffusions. Annals of Applied Probability, 24(4):1329-1374, 2014.

[27] J. G. Dai and R. J. Williams. Existence and uniqueness of semimartingale reflecting Brownian motions in convex polyhedrons. Theory of Probability and Its Applications, 40(1):1-40, 1995. Correctional note, 2006 (50), p. 346-347.

[28] P. Dupuis and R. J. Williams. Lyapunov functions for semimartingale reflecting Brownian motions. Annals of Probability, 22(2):680-702, 1994.

[29] J. M. Harrison and R. J. Williams. Multidimensional reflected Brownian motions having exponential stationary distributions. Annals of Probability, 15:115-137, 1987.

[30] J. A. Carillo, A. Jüngel, P. Markovich, G. Toscani, and A. Unterreiter. Entropy dissipation methods for degenerate parabolic problems and generalized Sobolev inequalities. Monatshefte für Mathematik, 133(1):1-82, 2001.

[31] S. A. Mohammed and M. K. R. Scheutzow. The stable manifold theorem for non-linear systems with memory: I. existence of the semiflow. Journal of Functional Analysis, 205:271-305, 2003

[32] O. Carbonell-Nicolau and R. McLean. Refinements of Nash equilibrium in potential games. Theoretical Economics, 9(3):555-582, 2014.

[33] D. Carfî and F. Musolino. A coopetetive-dynamical game model for currency markets stabilization. AAPP Physical, Mathematical, and Natural Sciences, 93(1):1-29, 2015.

[34] M. Campbell and L. Chayes. The isotropic O(3) model and the Wolff representation. Journal of Physics A, 31(13):L255-L259, 1998.

[35] M. Campbell and L. Chayes. Intermediate phases in mixed nematic/Heisenberg spin models. Journal of Physics A, 32(50):8881-8887, 1999.

[36] C. M. Fortuin, P. W. Kastelyn, and J. Ginibre. Correlation inequalities on some partially ordered sets. Communications in Mathematical Physics, 22:89-103, 1971.

[37] G. Grimmett. The Random-Cluster Model. Springer Verlag, 2006.

[38] J. Ginibre. General formulation of Griffiths' inequalities. Communications in Mathematical Physics, 16:310-328, 1970. 